\title{
Imperfectly informed voters and strategic extremism*
}

\author{
Enriqueta Aragonès ${ }^{\dagger}$ \\ Institut d'Anàlisi Econòmica-CSIC and Barcelona GSE
}

\author{
Dimitrios Xefteris ${ }^{\ddagger}$ \\ University of Cyprus
}

\section{October 2013}

\begin{abstract}
We analyze a unidimensional model of two-candidate electoral competition where voters have imperfect information about the candidates' policy proposals, that is, voters cannot observe the exact policy proposals of the candidates but only which candidate offers the most leftist/rightist platform. We assume that candidates are purely office motivated and that one candidate enjoys a valence advantage over the other. We characterize the unique Sequential Equilibrium in very-weakly undominated strategies of the game. In this equilibrium the behavior of the two candidates tends to maximum extremism, due to the voters' lack of information. But it may converge or diverge depending on the size of the advantage. For small values of the advantage candidates converge to the extreme policy most preferred by the median and for large values of the advantage candidates strategies diverge: each candidate specializes in a different extreme policy. These results are robust to the introduction of a proportion of well informed voters. In this case the degree of extremism decreases when the voters become more informed.
\end{abstract}

Keywords: Downsian model; imperfect information; advantaged candidate; maximum differentiation.

JEL Classification: D72

*Aragones acknowledges financial support by the Generalitat de Catalunya Grant number 2009 SGR 1126, the Spanish Ministry Education and Science ECO2012-37857 and the Barcelona Graduate School of Economics.

†Institut d'Anàlisi Econòmica, CSIC, Campus UAB, 08193 Bellaterra (Spain). Email: enriqueta.aragones@iae.csic.es

${ }^{\ddagger}$ Department of Economics, Faculty of Economics and Management, University of Cyprus. P.O. Box 20537, CY-1678 Nicosia (Cyprus). Email: xefteris.dimitrios@ucy.ac.cy 


\section{Introduction}

We introduce a new assumption in an otherwise standard Downsian model of two party electoral competition in order to account for the voters' lack of information about the electoral platforms proposed by the candidates. Specifically we assume that after both candidates announce simultaneously their policy positions, voters can only observe the announced platforms' relative positions on the policy space. That is, voters only know whether both candidates have chosen the same position, or if that is not the case, which candidate has chosen the most leftist or rightist position.

We show that with this modification the median voter result does not apply. In fact, our modified version of the standard Downsian model leads to some extreme and unexpected results. We find that when the voters' only information is the relative position of the candidates' choices on the policy space, the strategic response of the candidates is to choose the most extreme policies. Clearly, if there is any reason why candidates' may expect that being leftist offers an electoral advantage, all candidates will want to convince the voters that they are the most leftist ones and this will drive them to converge to an extreme position. However, when we break the symmetry of the candidates by introducing a small advantage for one of them, the strategic response of the candidates is still extreme but divergent: it drives each candidate's policy choice to a different end of the policy space.

The assumption that voters do not have as much information as candidates about the policy choices makes the model more realistic. In political science there exists a whole literature which tries to "estimate" the policy platforms of elected officials (see for example Mikhailov et al. 2012; Lowe et al. 2011; Benoit et al. 2009; Laver et al. 2003; Laver and Garry 2000). The existence of this literature alone is sufficient to back up the assumption that voters' information about candidates' policy platforms is far from being perfect. Moreover, Adams et al. (2011) document that voters react very poorly to parties' Left-Right policy shifts during an electoral campaign but very strongly to alterations in parties' policy images, which are usually shaped by interest groups endorsements. Notice that interest group endorsements offer to voters information only about the relative position of candidates, exactly as in this paper's working assumption. That is, the idea that the information that voters have when casting a ballot is not just imperfect but, also, mainly relative, seems to have a sound empirical support. Finally, it is worth

noting that many studies which try to estimate voters' awareness of American politics consider that a respondent is aware of candidates' differences if, given a specific policy issue, she can correctly identify which candidate, out of two, is more conservative (Layman and Carsey 2002). That is, the imperfect 
information that we consider in this paper that voters have about candidates' platforms is regarded enough by the aforementioned studies to characterize a voter as being aware of candidates' differences. In reality, no more than $80 \%$ of the voters can correctly determine which is the most conservative candidate in any given issue and any given election (Table 1, Layman and Carsey 2002). Hence, the imperfect information scenario that we consider here might be richer, not poorer, than the actual information that voters hold.

The particular set-up that we analyze requires the analysis of a game of imperfect information: voters cannot perfectly observe the strategies chosen by the candidates. We use the solution concept of sequential equilibrium (Kreps and Wilson 1982) and we apply a very mild refinement (Marx and Swinkels 1997) that focuses on undominated strategies to derive a unique equilibrium in terms of the candidates' strategies.

We assume that during the electoral campaign voters receive a signal that depends on the candidates' policy choices. If candidate A chooses a policy that is to the left of the policy chosen by candidate B, then the voters receive a signal that tells them only that candidate A's policy choice is more leftist than candidate B's, but they do not receive any information about the precise location of the candidates' policy choices, nor they know how close they are to their own ideal points. Similarly, if candidate A chooses a policy that is to the right of the policy chosen by candidate $B$, then the voters receive a signal that tells them only that: candidate A's policy choice is more rightist than candidate B's. Finally, if both candidates have chosen the same policy position, then the voters receive a signal that tells them exactly that, but they do not receive any information about the precise location of the candidates' policy choice. Thus, there are only three kinds of signals that the voter may receive: "candidate A is the most leftist", "candidate $\mathrm{A}$ is the most rightist", and "both candidates have exactly the same platform."

After receiving the signal, a voter has to compute the expected utility that she derives from each candidate, taking into account only her own beliefs about the candidates' choices given the observed signal. She will vote for the candidate from whom she obtains the largest expected utility. We find that, given the equilibrium choices of the candidates, a voter's optimal choice is always the following: if her ideal point is leftist (smaller than 1/2) then she votes for the most leftist candidate, and if her ideal point is rightist (larger than $1 / 2$ ) then she votes for the most rightist candidate. Thus, the behavior of the voter is not surprising at all given the kind of information available.

We also generalize the standard Downsian model by allowing one of the candidates to have a small valence advantage. In particular, we assume that when the voter is indifferent between the two candidates, 
she votes for candidate A with some fixed probability that is always equal or larger than $1 / 2$, and she votes for candidate B with the remaining probability (smaller or equal to $1 / 2$ ). The larger the probability bias that the voter holds for candidate $\mathrm{A}$ in case of indifference, the larger the advantage that candidate A enjoys.

We find that the size of the advantage plays a major role in determining the features of the equilibrium strategies. If the size of the advantage is small, that is, if the probability with which the indifferent voters vote for candidate $\mathrm{A}$ is close to $1 / 2$, then in equilibrium both candidates will choose policies that converge to one of the extremes of the policy space: the most leftist if the median voter is expected to have leftist ideal point, and the most rightist if the median voter is expected to have a rightist ideal point. In this equilibrium both candidates' use pure strategies.

However, for large values of the advantage, candidates' policy choices in equilibrium do not converge. In this case, if the median voter is expected to have a leftist ideal point, then in equilibrium the advantaged candidate will choose extreme leftist policies and the disadvantaged candidate will choose extreme rightist policies, whereas if the median voter is expected to have a rightist ideal point, then in equilibrium the advantaged candidate will choose extreme rightist policies and the disadvantaged candidate will choose extreme leftist policies. In this equilibrium both candidates use mixed strategies with full support that are symmetric with respect to each other. The density function corresponding to each equilibrium strategy is increasing with respect to the policy for one candidate and decreasing for the other, that is, in equilibrium each candidate assigns increasing amounts of probability to a different extreme of the policy space.

Thus, even though all the equilibria involve extreme policy positions, we find equilibria of two different kinds: convergent equilibria for small advantage sizes and divergent equilibria for large advantage sizes. The size of the valence advantage is considered small or large relative to the bias of the distribution of the ideal point of the median voter. That is, the larger the bias of the distribution of the median voter's ideal point, the larger the advantaged needed to have a divergent equilibrium. Notice that the relevance of the valence advantage to the voter's decision is always minimal (independently of its size) in the sense that it plays a role only when a voter is indifferent between the policy choices of the two candidates.

The intuition behind these results can be found combining the forces that make candidates choose divergent policies because one of them enjoys an advantage (for instance, as in Aragonès and Palfrey 2002 and Aragonès and Xefteris 2012), with those that make candidates choose extreme policies because voters will only react to some signals about their relative policy choices. When the candidates believe that the 
median voter has an extreme leftist ideal point, that is, when the uncertainty about the location of the median voter ideal point is small, then the effect of the advantage is reduced and the candidates' strategies in equilibrium are mainly driven by the aim to satisfy the median voter. In this case candidates' policy choices will tend to converge. Since the median voter will only react to their relative locations, they will converge to the extreme policy that is expected to be the most preferred by the median voter.

Otherwise, when uncertainty about the location of the median voter ideal point is large, candidates do not have a clear strategy that satisfies the median voter, and the effect of the advantage is back in place (even if the advantage only plays a role when the voter is indifferent policy-wise). The equilibrium in this case shows differentiation between candidates' platforms for the same reasons that explain equilibrium policy differentiation in the existing models of electoral competition with an advantaged candidate. In those models, the advantaged candidate chooses more centrist policies (preferred by the median voter), and the disadvantaged candidate chooses more extreme policies from both ends of the policy space (less preferred by the median voter). In our case, the fact that a voter only reacts to the relative position of the candidates, forces both candidates to choose extreme policies from different ends of the policy space, thus the differentiation in this case is extreme.

As in the existing literature on models with advantaged candidates, we find that in all the cases analyzed before, the candidate that enjoys the advantage obtains larger payoffs in equilibrium, and these payoffs increase with the size of the advantage. In addition, we find that when the advantage is large, the advantaged candidate's payoffs increase with the bias of the distribution of the ideal point of the median voter. Otherwise, if the distribution of the median voter's ideal point is symmetric, the candidates' equilibrium strategies are identical and the difference between the payoffs of the two candidates is very small. Regarding voters' welfare we find that voters are better off when the size of the advantage is larger (relative to the bias), because for larger advantage's sizes the expected policy outcome is less extreme.

Our results are computed on a discrete policy space (a finite number of equidistant policies). This assumption is irrelevant for the small advantage size case because the equilibrium is in pure strategies. But it is important for the large advantage case when the equilibrium is in mixed strategies. In this case, when the number of policies increases, the strategies of the candidates assign larger amounts of probability to extreme policies, and in the limit, when the number of policies approaches infinite, they converge to extreme and divergent pure strategies. Furthermore, as the number of policies increases the direct effect of the size of the advantage on the payoffs disappears, while its indirect effect (the size of the advantage compared to the probability that the median voter is leftist determines whether an equilibrium 
is convergent or not) still affects the probability of winning of each candidate. In the concluding section of the paper we argue that an extension of our results to a continuous policy space is straightforward.

We also compare our set-up and results to the existing models of electoral competition with an advantaged candidate where instead of defining the advantage as a larger probability of obtaining the vote from an indifferent voter, it is defined as an extra fixed amount of utility (Groseclose 2001). We find that the conventional model with an advantaged candidate can be embedded in our setup if we assume that the indifferent voter always votes for the advantaged candidate (probability one). Since we cover a larger set of possibilities, our model is a generalization of the conventional one.

Finally, we check the robustness of the informational setting by introducing a proportion of well informed voters ${ }^{1}$. We show that increasing the proportion of informed voters reduces the extremism of the candidates' strategies and increases voters' welfare. We also find that introducing small enough proportions of informed voters in our otherwise misinformed electorate we obtain equilibrium strategies that are very similar to the ones described above and as the proportion of informed voters approaches zero the equilibrium strategies converge to the ones corresponding to the setup with a complete misinformed electorate. We also show that the degree of extremism of the policy outcome increases with the voters' lack of information.

The rest of the paper is organized as follows. In the next section we relate our results to the existing literature. In Section 3 we describe the formal model. Section 4 is devoted to characterize the equilibria. In section 5 we discuss the features of the equilibria. Section 6 compares the results obtained here with those from the conventional model of an advantage candidate and Section 7 concludes.

\section{Related literature}

The assumption that voters only observe the relative position of candidates in the policy space is not new in the economics and political science literature. The paper most closely related to the informational set-up of our paper is one by McKelvey and Ordeshook (1985). Like us, they also consider that voters only have relative information about candidates' platforms but, unlike us, they consider that voters have very good information about the policy preferences of their fellow citizens (each voter knows exactly how many voters have ideal policies to the left of her ideal policy and how many voters have ideal policies to

\footnotetext{
${ }^{1}$ Alternatively, this assumption could be interpreted as if, with some probability, all voters are correctly informed about the candidates policy choices.
} 
the right of her ideal policy) and that each voter believes that all other voters are perfectly informed about the policy platforms of the candidates. That is, they withdraw some information from one dimension of the standard Downsian model (awareness of the exact location of candidates' platforms) while they add some information on another dimension of the model (distribution of fellow citizens' policy preferences) and some extra behavioral assumptions (belief that everybody else is perfectly informed) and they show that the median voter result is robust to this specification. Our analysis will demonstrate that, without the aforementioned informational "additions" of McKelvey and Ordeshook (1985), relative information about candidates' platforms leads to policy choices far from the median.

Another paper that is related to ours is one by Gul and Pesendorfer (2009) who consider the possibility of voters being ignorant about the policy choice of a candidate in a binary policy framework with heterogeneous (in terms of non-policy characteristics) candidates. They consider that voters are homogeneous in terms of policy preferences and heterogeneous in terms of which candidate's non-policy characteristics they value more, and they show that candidate differentiation could be encountered in the equilibrium of this model. That is, they also argue that lack of information about candidate platforms may result in non-convergent candidate behavior when candidates are heterogeneous. The binary nature of their policy space, though, cannot provide answers on whether this lack of information leads to moderate candidate behavior (only mild differentiation around some notion of a center of the policy space) or to completely extremist behavior. Moreover, the assumption that all voters are homogeneous in terms of policy preferences makes their analysis depart to a great extent from the standard Downsian framework of analysis. Our results reinforce their findings (in the sense that policy differentiation could be due to lack of voters' information about candidates' policy platforms), and complement them by establishing that differentiation, whenever it occurs, is (nearly) maximal.

Our results also offer new arguments in favor of the standard theory of voting (a voter's utility from a certain policy is decreasing in the distance between this policy and her ideal policy) when compared to the, so-called, theory of directional voting (see for example Rabinowitz and Macdonald 1989). In brief, directional voting assumes that a voter's utility from a certain policy is decreasing (increasing) in the distance between this policy and the left extreme when the voter's ideal policy is located in the left (right) half of the policy axis. The assumptions of these models are, obviously, incompatible with basic rational behavior assumptions but their proponents argue that they exhibit a better fit with real elections observations (they predict non-moderate candidate behavior) than the standard model (which predicts moderate candidate behavior). ${ }^{2}$ In this paper we consider a variation of the standard Downsian model

\footnotetext{
${ }^{2}$ One should stress here that there is no consensus in empirical literature that directional voting fits with the data better
} 
(we just replace the assumption of perfect information about candidates' platforms with one of imperfect information) and we show that non-moderate candidate behavior (convergent or divergent) is the most reasonable outcome. Hence, the alleged "better-fit" of the directional voting theory with the data when compared to the standard theory of voting need not be due to voters actually behaving in the unintuitive manner that directional voting considers but due to the fact that the information that voters hold is imperfect.

Theoretical literature based on the standard Downsian model (at least as far as voters' behavior is concerned) offers various alternative explanations for the observed non-moderate candidate platform choices in two-candidate elections. Palfrey (1984) considers the variation of the standard model in which the two candidates face the potential entry of a third candidate and he shows that this makes the two main candidates differentiate to a great extent. Roemer (1994), in the context of the Calvert (1985) and Wittman (1977) variation of the Downsian model (candidates with policy preferences), shows that when candidates are imperfectly informed about voters' preferences, non-moderate and divergent behavior can be also encountered. Finally, Grosser and Palfrey (2013) show that extremist equilibria arise in the citizen-candidate model when players' ideal policies are private information while Glaeser et al. (2005) suggest that the observed extremist positioning of candidates can be attributed to the fact that candidates need to induce their core constituencies to actively support them (or to make donations) and, hence, they deviate away from moderate behavior. The present paper complements this literature by offering a new explanation.

\section{The model}

There are two candidates, $A$ and $B$, that compete in an election. The policy space $X$ is the set of $n$ points on the $[0,1]$ interval, $x_{k}=\frac{k-1}{n-1}, k=1,2, \ldots, n$. Before the election, candidates simultaneously choose policy platforms $x_{A}$ and $x_{B}$ respectively from the policy space $X$. Each candidate's objective is to maximize his probability of winning the election. We assume full commitment of the candidates to the chosen platforms.

Without loss of generality, we assume that there is a unique voter who has preferences over policies represented by a utility function $u_{m}(x)=\phi\left(\left|x-x_{m}\right|\right)$ where $\phi(\cdot)$ is strictly decreasing with respect to $\left|x-x_{m}\right|$ and $x_{m} \in X$ represents the voter's ideal policy.

than standard theory of voting. See for example Tomz and Van Houweling (2008). 
The voter cannot observe the policies chosen by the candidates, $\left(x_{A}, x_{B}\right)$, but only a signal that refers to the relative location of the policies chosen by the candidates, that is, the voter only knows whether the policies chosen by the two candidates are the same or they are different, and in this case, which policy is more rightist and which one is more leftist. Formally, the signal that the voter receives after the candidates choose their platforms is denoted by $S \in\{L, R, I\}$ and is defined as follows:

$$
S\left(x_{A}, x_{B}\right)=\left\{\begin{array}{ccc}
L & \text { if } & x_{A}<x_{B} \\
I & \text { if } & x_{A}=x_{B} \\
R & \text { if } & x_{A}>x_{B}
\end{array}\right.
$$

The voter has beliefs over the candidates' chosen policies $\left(x_{A}, x_{B}\right)$ that depend on the observed signal $S\left(x_{A}, x_{B}\right)$. The beliefs of the voter are represented by a probability distribution over $X^{2}$ and are assumed to be common knowledge by the candidates.

For $S\left(x_{A}, x_{B}\right)=L$ let $b_{L}$ denote the density function of the probability distribution over the set

$$
Y_{L}=\left\{\left(x_{A}, x_{B}\right) \in X^{2}: x_{A}<x_{B}\right\}
$$

$b_{L}$ represents the beliefs of the voter regarding the pairs of policies chosen by the candidates when she observes signal $L$. Similarly, for $S\left(x_{A}, x_{B}\right)=R$ let $b_{R}$ denote the density function of the probability distribution over the set

$$
Y_{R}=\left\{\left(x_{A}, x_{B}\right) \in X^{2}: x_{A}>x_{B}\right\}
$$

$b_{R}$ represents the beliefs of the voter regarding the pairs of policies chosen by the candidates when she observes signal $R$. Finally, for $S\left(x_{A}, x_{B}\right)=I$ let $b_{I}$ denote the density function of the probability distribution over the set

$$
Y_{I}=\left\{\left(x_{A}, x_{B}\right) \in X^{2}: x_{A}=x_{B}\right\}
$$

$b_{I}$ represents the beliefs of the voter regarding the pairs of policies chosen by the candidates when she observes signal $I$. See figure $1 \mathrm{a}$ and $1 \mathrm{~b}$. 
[Insert Figures 1a, $1 \mathrm{~b}$ and $1 \mathrm{c}]$

It is possible that the beliefs of the voter after receiving a given signal assign positive probability to only a proper subset of the set of pairs of policies consistent with the observed signal. Figure 1c shows an example of such a case. For any signal $S$, let $y_{S}\left(b_{S}\right)$ denote the support of $b_{S}$, that is, the subset of $Y_{S}$ that contains all pairs of policies to which $b_{S}$ assigns positive probability. The beliefs of the voter over the candidates' strategies after observing each signal are common knowledge for all players.

Formally, we have that the voter's expected utility from candidate $A$ 's policy proposal $\bar{x}_{A}$ when candidate $B$ 's proposal is $\bar{x}_{B}$, after receiving signal $S=S\left(\bar{x}_{A}, \bar{x}_{B}\right)$ is given by

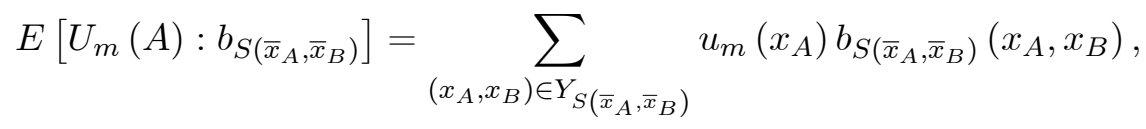

The voter decides to vote for candidate $A$ if

$$
E\left[U_{m}(A): b_{S\left(\bar{x}_{A}, \bar{x}_{B}\right)}\right]>E\left[U_{m}(B): b_{S\left(\bar{x}_{A}, \bar{x}_{B}\right)}\right]
$$

which holds if and only if

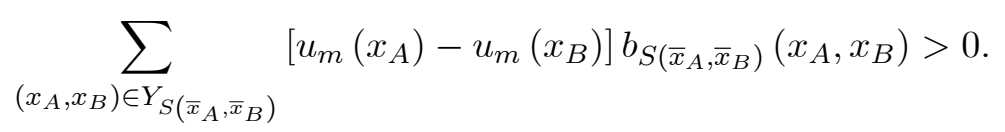

Notice that since $Y_{I}=\left\{\left(x_{A}, x_{B}\right) \in X^{2}: x_{A}=x_{B}\right\}$ when $S=I$ we have any belief that the voter might have has to imply that $x_{A}=x_{B}$, and thus we have that for all $b_{I} \in \Delta X^{2}$.

$$
E\left[U_{m}(A): b_{I\left(x_{A}, x_{B}\right)}\right]=E\left[U_{m}(B): b_{I\left(x_{A}, x_{B}\right)}\right]
$$

because

$$
\sum_{\left(x_{A}, x_{B}\right) \in Y_{I}}\left[u_{m}\left(x_{A}\right)-u_{m}\left(x_{B}\right)\right] b_{I}\left(x_{A}, x_{B}\right)=0
$$


In particular, we have that if the voter's beliefs after each signal are represented by a uniform probability over a full support then it is easy to see that a voter with $x_{m}<\frac{1}{2}$ will strictly prefer to vote for $A$ when the signal is $L$ and will strictly prefer to vote for $B$ when the signal is $R$. Similarly, with uniform beliefs over a full support a voter with $x_{m}>\frac{1}{2}$ will strictly prefer to vote for $B$ when the signal is $L$ and will strictly prefer to vote for $A$ when the signal is $R$.

Instead, if the beliefs are not uniform or they do not have full support then the voter's optimal decision is not so obvious and it depends on the specific functional forms of the voter's beliefs.

We assume that candidates do not know the exact location of $x_{m}$ and they have beliefs about it that are common knowledge and are represented by a probability distribution $F: X \rightarrow[0,1]$ with full support over $X$ and with density $f: X \rightarrow[0,1]$. If candidates knew the exact value of $x_{m}$, they could anticipate the probability with which the voter votes for each candidate and they could use it to decide their optimal strategy. Since we assume that candidates have beliefs about the value of $x_{m}$, they can only anticipate the expected probability with which the voter votes for each candidate. ${ }^{3}$ Let $p_{S\left(x_{A}, x_{B}\right)}\left(F, b_{S\left(x_{A}, x_{B}\right)}\right)$ denote the candidates' expected probability with which the voter votes for candidate $A$ after observing signal $S\left(x_{A}, x_{B}\right)$. Then we have that:

$$
p_{S\left(x_{A}, x_{B}\right)}\left(F, b_{S\left(x_{A}, x_{B}\right)}\right)=\sum_{i=1}^{n} a_{S\left(x_{A}, x_{B}\right)}\left(x_{i}, b_{S\left(x_{A}, x_{B}\right)}\right) f\left(x_{i}\right)
$$

where the function $a_{S\left(x_{A}, x_{B}\right)}\left(x_{m}, b_{S\left(x_{A}, x_{B}\right)}\right)$ denotes the probability with which the voter votes for candidate $A$ after she observes signal $S$, when her ideal point is $x_{m}$, and the beliefs induced by signal $S$ are $b_{S\left(x_{A}, x_{B}\right)}$. Formally, the function $a_{S\left(x_{A}, x_{B}\right)}\left(x_{m}, b_{S\left(x_{A}, x_{B}\right)}\right)$ is defined as follows:

$$
a_{S\left(x_{A}, x_{B}\right)}\left(x_{m}, b_{S\left(x_{A}, x_{B}\right)}\right)=\left\{\begin{array}{lll}
1 & \text { if } & E\left[u_{m}\left(x_{A}\right): b_{S\left(x_{A}, x_{B}\right)}\right]>E\left[u_{m}\left(x_{B}\right): b_{S\left(x_{A}, x_{B}\right)}\right] \\
\rho & \text { if } & E\left[u_{m}\left(x_{A}\right): b_{S\left(x_{A}, x_{B}\right)}\right]=E\left[u_{m}\left(x_{B}\right): b_{S\left(x_{A}, x_{B}\right)}\right] \\
0 & \text { if } & E\left[u_{m}\left(x_{A}\right): b_{S\left(x_{A}, x_{B}\right)}\right]<E\left[u_{m}\left(x_{B}\right): b_{S\left(x_{A}, x_{B}\right)}\right]
\end{array} .\right.
$$

\footnotetext{
${ }^{3}$ Our assumptions offer an alternative interpretation: consider infinite voters with ideal points distributed on the policy space according to the function $F$; candidates have complete information about the distribution of the voters' ideal points and maximize their vote share, instead of their probability of winning.
} 
where $\rho \in\left[\frac{1}{2}, 1\right]$. Thus, we assume that the voter votes for the party whose platform offers her the largest utility ${ }^{4}$, given a fixed value of $x_{m}$. If the beliefs of the voter after observing signal $S$ are such that the policy proposal of candidate $A$ offers higher utility to the voter, in expected terms, than the policy proposal of candidate $B$, then the voter will vote for candidate $A$ after observing signal $S$, and thus we will have that $a_{S\left(x_{A}, x_{B}\right)}\left(x_{m}, b_{S\left(x_{A}, x_{B}\right)}\right)=1$. Similarly, if the beliefs of the voter after observing signal $S$ are such that the policy proposal of candidate $B$ offers higher utility to the voter, in expected terms, than the policy proposal of candidate $A$, then the voter will vote for candidate $B$ after observing signal $S$, and thus we will have that $a_{S\left(x_{A}, x_{B}\right)}\left(x_{m}, b_{S\left(x_{A}, x_{B}\right)}\right)=0$. Finally, if the beliefs of the voter after observing signal $S$ are such that the policy proposal of candidate $A$ offers equal utility to the voter, in expected terms, as the policy proposal of candidate $B$, then after observing signal $S$ the voter will vote for candidate $A$ with probability $\rho \in\left[\frac{1}{2}, 1\right]$ and she will vote for candidate $B$ with probability $1-\rho$, thus we have that $a_{S\left(x_{A}, x_{B}\right)}\left(x_{m}, b_{S\left(x_{A}, x_{B}\right)}\right)=\rho$.

Notice that the probability $\rho$ introduces an asymmetry between the two candidates that implies an advantage for candidate $A .^{5}$ The effect of this advantage on the voter's decision is always minimal since it only plays a role when the median voter is indifferent. Even though the size of the advantage only affects the probability with which a policy-wise indifferent voter chooses candidate $A$ over candidate $B$, it has non trivial implications over the policy outcomes: it induces candidates to choose divergent policies in equilibrium, in particular the advantaged candidate will tend to play more centrist policies and the disadvantaged candidate will tend to play more extreme policies. The functional form of the voters' utility function assumed in this paper can be thought of as an extension of the one used in the model of electoral competition in Aragonès and Palfrey (2002). In that case, the indifferent voter was assumed to vote for one of the candidates with probability one and that was the advantaged candidate. Here we assume that when the voter is indifferent she votes for each candidate with a different probability and different from $1 / 2$ as well. In our case the advantaged candidate is the one that is chosen by the indifferent voter with the largest probability.

The timing of the game is as follows. First both candidates choose their policy platforms simultaneously. We consider both pure and mixed strategies for the candidates. Given the candidates policy

\footnotetext{
${ }^{4}$ If the voter had perfect information she would vote for candidate $A$ whenever $\left(x_{A}, x_{B}\right)$ is such that $\left|x_{A}-x_{m}\right|<$ $\left|x_{B}-x_{m}\right|$; she would vote for candidate $B$ whenever $\left(x_{A}, x_{B}\right)$ is such that $\left|x_{A}-x_{m}\right|>\left|x_{B}-x_{m}\right|$, and whenever $\left(x_{A}, x_{B}\right)$ is such that $\left|x_{A}-x_{m}\right|=\left|x_{B}-x_{m}\right|$ she would vote for candidate A with probability $\rho$ and for candidate B with probability $1-\rho$.

${ }^{5}$ Under the alternative interpretation in which candidates have complete information about the voters' ideal points and maximize their vote share instead of their probability of winning, the advantage can be interpreted as follows: there is a proportion $\rho$ of indifferent voters that vote for candidate $A$ and a proportion $1-\rho$ of indifferent voters that vote for candidate B.
} 
choices, a signal is sent to the voter. This signal will tell the voter that candidate $A$ has chosen a policy to the left of candidate $B$ 's policy choice (formally, signal $L$ ), or it will tell the voter that candidate $A$ has chosen a policy to the right of candidate $B$ 's policy choice (formally, signal $R$ ), or it will tell the voter that both candidates have chosen the same policy (formally, signal $I$ ).

If candidates are using pure strategies, the signal that the voter receives is based on the candidates' choice of policies described by the pure strategies. If candidates are using mixed strategies, the signal that the voter receives is based on the realization of the candidates' choice of policies described by the mixed strategies.

The beliefs of the voter over the candidates' policy choices after receiving each signal are given. The voter evaluates the utility that she derives from each candidate taking into account the signal received and the beliefs corresponding to that signal, and she votes for the candidate that offers her a higher expected utility. Notice that the voter's prior beliefs do not have to be updated during the game, because the voter does not acquire any new information that may affect her beliefs about the candidates' choices after observing a given signal. Thus the beliefs that the voter uses to interpret each signal, and to evaluate each candidate, coincide with the beliefs of the voter at the beginning of the game and are common knowledge to all players. In particular, we have that for all $S \in\{L, R, I\}$ the functions $p_{S}\left(F, b_{S}\right)$ are common knowledge to the candidates when they make their strategy choices.

Let $\left(x^{A}, x^{B}\right) \in X^{2}$ denote a pair of pure strategies for candidates $A$ and $B$ respectively. Let

$$
\Pi^{A}\left(x^{A}, x^{B} \mid b_{L}, b_{I}, b_{R}\right)=p_{S\left(x^{A}, x^{B}\right)}\left(F, b_{S\left(x^{A}, x^{B}\right)}\right)
$$

and

$$
\Pi^{B}\left(x^{A}, x^{B} \mid b_{L}, b_{I}, b_{R}\right)=1-p_{S\left(x^{A}, x^{B}\right)}\left(F, b_{S\left(x^{A}, x^{B}\right)}\right)
$$

denote the payoffs of candidates $A$ and $B$ respectively when their policy choices are $\left(x^{A}, x^{B}\right)$ and the voter's beliefs are $b_{L}, b_{I}, b_{R}$.

Let $\left(\sigma^{A}, \sigma^{B}\right)$ denote a pair of mixed strategies for candidates $A$ and $B$ respectively, where $\sigma^{i}=$ $\left(\sigma_{1}^{i}, \ldots, \sigma_{n}^{i}\right) \in \Delta X$ denotes the probability with which candidate $i$ chooses each policy. Let 


$$
\Pi^{A}\left(\sigma^{A}, \sigma^{B} \mid b_{L}, b_{I}, b_{R}\right)=\sum_{S \in\{L, I, R\}} \sum_{\left(x^{A}, x^{B}\right) \in Y_{S}} p_{S\left(x^{A}, x^{B}\right)}\left(F, b_{S\left(x^{A}, x^{B}\right)}\right) \sigma^{A}\left(x^{A}\right) \sigma^{B}\left(x^{B}\right)
$$

and

$$
\Pi^{B}\left(\sigma^{A}, \sigma^{B} \mid b_{L}, b_{I}, b_{R}\right)=1-\Pi^{A}\left(\sigma^{A}, \sigma^{B} \mid b_{L}, b_{I}, b_{R}\right)
$$

denote the payoffs of candidates $A$ and $B$ respectively when their strategies are $\left(\sigma^{A}, \sigma^{B}\right)$ and the voter's beliefs are $b_{L}, b_{I}, b_{R}$.

The extensive game described above is a game of imperfect information because the voter cannot observe the strategies chosen by the candidates, and it is also a game of incomplete information because the candidates cannot observe the type of the voter. Notice, that, in game-theoretical terms, each of the three signals corresponds to a distinct information set. This is why in the remainder we use the terms "signal" and "information set" interchangeably.

In order to solve it we use the equilibrium concept of Sequential Equilibrium (SE). Given the functions $S\left(x_{A}, x_{B}\right)$ and $a_{s}\left(x_{m}, b_{S}\right)$ for all $S \in\{L, R, I\}$, and given the candidates' common beliefs on the voter's ideal point, $F$, a Sequential Equilibrium is described by two ${ }^{6}$ (possibly) mixed strategies

$$
\sigma^{* A}=\left(\sigma_{1}^{* A}, \ldots, \sigma_{n}^{* A}\right) \in \Delta X \text { and } \sigma^{* B}=\left(\sigma_{1}^{* B}, \ldots, \sigma_{n}^{* B}\right) \in \Delta X
$$

and voter's beliefs

$$
b_{S} \text { for all } S \in\{L, R, I\}
$$

such that

$$
\Pi^{A}\left(\sigma^{* A}, \sigma^{B} \mid b_{L}, b_{I}, b_{R}\right) \geq \Pi^{A}\left(\sigma^{* A}, \sigma^{* B} \mid b_{L}, b_{I}, b_{R}\right) \geq \Pi^{A}\left(\sigma^{A}, \sigma^{* B} \mid b_{L}, b_{I}, b_{R}\right)
$$

\footnotetext{
${ }^{6}$ This is without loss of generality. Formally, in this setup a SE should include $2+3 \times n$ strategies: one strategy for each candidate and one strategy for the voter for each possible ideal policy of the voter in all three information sets. A belief system, though, uniquely defines the optimal strategy of the voter for each possible ideal policy and each information set. Hence, including both the beliefs and the strategies of the voter is unnecessary and just makes notation heavier.
} 
for all $\sigma^{A}$ and $\sigma^{B}$ given $\left(\sigma^{* A}, \sigma^{* B}\right)$ and $\left(b_{L}, b_{I}, b_{R}\right)$

and there is a sequence of completely mixed strategies $\left(\sigma_{j}^{A}, \sigma_{j}^{B}\right)_{j=1}^{\infty}$ with $\lim _{j \rightarrow \infty}\left(\sigma_{j}^{A}, \sigma_{j}^{B}\right)_{j=1}^{\infty}=$ $\left(\sigma^{* A}, \sigma^{* B}\right)$ such that $\left(b_{L}, b_{I}, b_{R}\right)=\lim _{j \rightarrow \infty}\left(b_{L}, b_{I}, b_{R}\right)_{j}$ where $\left(b_{L}, b_{I}, b_{R}\right)_{j}$ denotes the beliefs derived from strategy profile $\left(\sigma_{j}^{A}, \sigma_{j}^{B}\right)$ using Bayes' rule (sequential rationality). In case $\sigma^{* A}$ and $\sigma^{* B}$ are fully mixed, it follows that

$$
\begin{gathered}
b_{L}\left(x_{i}, x_{i^{\prime}}\right)=\frac{\sigma_{i}^{* A} \sigma_{i^{\prime}}^{* B}}{\sum_{k=1}^{n-1}\left[\sigma_{k}^{* A} \sum_{j=k+1}^{n} \sigma_{j}^{* B}\right]} \text { for }\left(x_{i}, x_{i^{\prime}}\right) \in Y_{L}, \\
b_{I}\left(x_{i}, x_{i^{\prime}}\right)=\frac{\sigma_{i}^{* A} \sigma_{i^{\prime}}^{* B}}{\sum_{k=1}^{n} \sigma_{k}^{* A} \sigma_{k}^{* B}} \text { for }\left(x_{i}, x_{i^{\prime}}\right) \in Y_{I} \\
b_{R}\left(x_{i}, x_{i^{\prime}}\right)=\frac{\sigma_{i}^{* A} \sigma_{i^{\prime}}^{* B}}{\sum_{k=1}^{n-1}\left[\sigma_{k}^{* B} \sum_{j=k+1}^{n} \sigma_{j}^{* A}\right]} \text { for }\left(x_{i}, x_{i^{\prime}}\right) \in Y_{R} .
\end{gathered}
$$

Notice that each belief system $\left(b_{L}, b_{I}, b_{R}\right)$ induces a normal form constant-sum game between the two candidates. We refer to it as the reduced game given the belief system $\left(b_{L}, b_{I}, b_{R}\right)$. A reduced game between the two candidates is considered to be trivial when each candidate is indifferent among all available strategies independently of what his opponent candidate is expected to play.

In order to rule out equilibria with belief systems which induce a trivial reduced game between the two candidates we employ the very-weak-dominance notion of Marx and Swinkels (1993). That is, we consider that a strategy $\sigma^{A}$ is undominated given $\left(b_{L}, b_{I}, b_{R}\right)$ if there exists no $\stackrel{\circ}{\sigma}^{A}$ such that $\Pi^{A}\left(\stackrel{\circ}{\sigma}^{A}, \sigma^{B} \mid b_{L}, b_{I}, b_{R}\right) \geq \Pi^{A}\left(\sigma^{A}, \sigma^{B} \mid b_{L}, b_{I}, b_{R}\right)$ for all $\sigma^{B}$ (the equivalent holds for player $B$ ). We only consider those SE in which the strategies of the two candidates are undominated strategies.

We characterize the unique strategies of the candidates in a sequential equilibrium in undominated strategies for an even number of policies. When the number of policies is odd the analysis is analogous and we offer some details of it in Appendix 2. For the main results we assume that $F\left(x_{n / 2}\right) \geq \frac{1}{2}$. The analysis of the case $F\left(x_{n / 2}\right) \leq \frac{1}{2}$ is symmetric to this one and it is discussed but nor formalized. We show that there exists a unique sequential equilibrium in undominated strategies for any $\rho \in\left[\frac{1}{2}, 1\right]$, except for the very special case that $\rho=F\left(x_{n / 2}\right)=\frac{1}{2}$ in which all equilibria are in dominated strategies. 


\section{Characterization of the Equilibria}

There are the three unique possibilities of the candidates' expected payoff in equilibrium. Observe that in a SE one of the following should hold: a) $p_{L}=p_{R}=\rho$, b) $p_{L} \neq p_{R}$ and $\rho \in\left[\min \left\{p_{L}, p_{R}\right\}, \max \left\{p_{L}, p_{R}\right\}\right]$, and c) $\rho>\max \left\{p_{L}, p_{R}\right\}$ or $\rho<\min \left\{p_{L}, p_{R}\right\}$. Here and for the rest of the paper we write $p_{S}$ to denote $p_{S}\left(F, b_{S}\right)$ for $S \in\{L, R, I\}$. We will analyze the conditions under which a $\mathrm{SE}$ in undominated strategies exists in each one of these cases and the features of such equilibria. The next proposition sheds some light on the kind of equilibria that we will encounter.

Proposition 1 a) There is no $S E$ in undominated strategies in which $p_{L}=p_{R}=\rho$. b) If in a SE in undominated strategies we have $p_{L} \neq p_{R}$ and $\rho \in\left[\min \left\{p_{L}, p_{R}\right\}, \max \left\{p_{L}, p_{R}\right\}\right]$ then candidates converge to an extreme policy. c) Otherwise, $\left(\rho>\max \left\{p_{L}, p_{R}\right\}\right.$ or $\left.\rho<\min \left\{p_{L}, p_{R}\right\}\right)$ in a SE in undominated strategies candidates play mixed strategies with full support.

All proofs can be found in Appendix 1.

The above proposition does not guarantee existence of SE in undominated strategies for any parameter values. It just gives properties of such equilibria if they exist. It is easy to see that if $p_{L}=p_{R}=\rho$ no candidate has an undominated strategy, because any equilibrium strategy is very-weakly-dominated by any other strategy. Next we offer complete characterizations of the equilibria that exist for all the other cases and we show that for any set of parameter values there is an essentially unique SE in undominated strategies. By essentially unique we mean that the candidates' equilibrium strategies are uniquely defined, but there might be multiple belief systems that support the same strategy profile.

We start assuming that $F\left(x_{n / 2}\right)>\frac{1}{2}$, that is, candidates' expect the ideal point of the median voter to be rather leftist. The case $F\left(x_{n / 2}\right)<\frac{1}{2}$ is very similar and we only offer some comments about it. Afterwards, we derive the equilibrium results corresponding to $F\left(x_{n / 2}\right)=\frac{1}{2}$.

First, suppose that the size of the advantage is small relative to the asymmetry in the expected location of the median voter's ideal point, that is, suppose that $F\left(x_{n / 2}\right) \geq \rho$. We find that the equilibrium is such that $\rho \in\left[\min \left\{p_{L}, p_{R}\right\}, \max \left\{p_{L}, p_{R}\right\}\right]$ and there exists an essentially unique SE in undominated strategies

such that both parties converge to an extreme of the policy space. When $F\left(x_{n / 2}\right)>\frac{1}{2}$, that is, when the ideal point of the voter is most likely to be to the left, in a SE we cannot have candidates expecting the voter to vote with larger probability for a candidate whose proposed policy is the most rightist. Thus, in 
this case both candidates have strong incentives to propose leftist policies, and since the voter will only be aware of the relative position of the candidates' choices in the policy space, their choices will converge to the most extreme leftist policy. In this case we have existence of an essentially unique SE in undominated strategies.

Proposition 2 An essentially unique SE in undominated strategies exists and it is such that the candidates' strategies are $\left(x^{A}, x^{B}\right)=(0,0)$ if and only if $F\left(x_{n / 2}\right) \geq \rho$.

Observe that in this equilibrium we will have that $p_{L}=\rho \leq F\left(x_{n / 2}\right)$ and $p_{R}=1-\rho \geq 1-F\left(x_{n / 2}\right)$. That is, since in equilibrium both candidates choose the same policy location, their winning probability is given by the asymmetric tie breaking rule used by the voter. When the size of the advantage is relatively small, the disadvantaged candidate does not have strong incentives to differentiate, and it allows for the possibility of a convergent equilibrium. This equilibrium collapses for larger values of $\rho$, because the probability with which candidate $B$ wins, if he chooses the same policy platform as that of candidate $A$, becomes much smaller. In particular when $\rho>F\left(x_{n / 2}\right)$, candidate $B$ prefers to differentiate and obtain $p_{R}=1-F\left(x_{n / 2}\right)>1-\rho$ rather than offer the same policy with $A$ and get $1-\rho$ probability of being elected.

Notice that if we assume that $F\left(x_{n / 2}\right)<\frac{1}{2}$, then it would not be possible to have $p_{R} \leq \rho \leq p_{L}$ in equilibrium, that is, if the ideal point of the voter is most likely to be to the right, in equilibrium we cannot have candidates expecting the voter to vote with larger probability for a candidate whose proposed policy is more leftist. In this case, we would have that the essentially unique SE in undominated strategies should satisfy $p_{L} \leq \rho \leq p_{R}$ and to be such that both candidates converge to the right extreme of the policy space.

Now we consider the case in which the size of the advantage is large relative to the asymmetry in the expected location of the median voter's ideal point, that is, suppose that $F\left(x_{n / 2}\right)<\rho$. The next proposition shows that for these high values of $\rho$ there exists a unique SE in undominated strategies. In this case, the asymmetry between the candidates, defined by the size of the advantage, has a larger effect on the equilibrium strategies, and induces the candidates to use mixed strategies: the disadvantaged candidate is better off mixing, otherwise the advantage candidate would imitate his policy choices and drive his probability of winning down to $1-\rho$. We find that in this equilibrium both candidates will use mixed strategies with full support. 
Proposition $3 A$ unique SE in undominated strategies exists and it is such that the candidates' strategies are $\sigma_{k}^{* A}=\left(H^{*}\right)^{k-1} \frac{1-H^{*}}{1-\left(H^{*}\right)^{n}}$ and $\sigma_{k}^{* B}=\left(H^{*}\right)^{n-k} \frac{1-H^{*}}{1-\left(H^{*}\right)^{n}}$ for $k=1, \ldots, n$ with $H^{*}=\frac{\rho-F\left(x_{n / 2}\right)}{\rho-1+F\left(x_{n / 2}\right)}<1$ and $b_{L}\left(x_{i}, x_{i^{\prime}}\right)=\frac{\left(H^{*}\right)^{n+i-i^{\prime}-1}\left(1-H^{*}\right)^{2}}{1-n\left(H^{*}\right)^{n-1}}, b_{I}\left(x_{i}, x_{i^{\prime}}\right)=\frac{\left(H^{*}\right)^{i-i^{\prime}}}{n}, b_{R}\left(x_{i}, x_{i^{\prime}}\right)=\frac{\left(H^{*}\right)^{i-i^{\prime}-1}\left(1-H^{*}\right)^{2}}{n-1-H^{*}+\left(H^{*}\right)^{n}}$ if and only if $F\left(x_{n / 2}\right)<\rho$.

Observe that in this equilibrium we have that $p_{L}=F\left(x_{n / 2}\right)<\rho$ and $p_{R}=1-F\left(x_{n / 2}\right)>1-\rho$, that is, the advantaged candidate expects to win with larger probability than the disadvantaged candidate, but in this case the probabilities of winning are not determined by the size of the advantage, instead they are mainly determined by the expected location of the median voter's ideal point. We have that $\frac{\partial \sigma_{k}^{* A}}{\partial k}=\frac{\left(H^{*}\right)^{k-1}\left(1-H^{*}\right)}{1-\left(H^{*}\right)^{n}} \ln H^{*}<0$ and $\frac{\partial \sigma_{k}^{* B}}{\partial k}=-\frac{\left(H^{*}\right)^{n-k}\left(1-H^{*}\right)}{1-\left(H^{*}\right)^{n}} \ln H^{*}>0$ since $H^{*}<1$, thus $\sigma_{k}^{* A}$ decreases with $k$ and $\sigma_{k}^{* B}$ increases with $k$. Figures $2 \mathrm{a}, 2 \mathrm{~b}, 2 \mathrm{c}$ and $2 \mathrm{~d}$ present examples of such mixed equilibria.

[Insert Figures 2a, 2b, 2c and 2d about here]

Notice that the equilibrium strategies put increasing amounts of probability on policies that are at the extremes of the policy space and candidates differentiate: each candidate's strategy assigns most of the probability to a different extreme of the policy space. The candidates' equilibrium strategies are symmetric to each other. The advantaged candidate's equilibrium strategy assigns most of its probability to policies that are on the left side of the policy space, while the disadvantaged candidate's equilibrium strategy assigns most of its probability to policies that are on the right side of the policy space. The reason is that both candidates believe that it is more likely that the ideal point of the median voter is on the left side of the policy space $\left(F\left(x_{n / 2}\right)>\frac{1}{2}\right)$. This leads the advantaged candidate to assign large amounts of probability to policies that are close to zero, and thus, in order to differentiate, the disadvantaged candidate has to assign most of his probability to policies that are close to one.

We find that in equilibrium the voter votes for $A$ as follows: with probability one if $x_{m}<\frac{1}{2}$ and we are in information set $L$, with probability one if $x_{m}>\frac{1}{2}$ and we are in information set $R$, and with probability $\rho$ if we are in information set $I$. Otherwise, the voter votes for $B$.

If instead we had assumed that $F\left(x_{n / 2}\right)<\frac{1}{2}$, we would have that $p_{L}=F\left(x_{n / 2}\right)<\frac{1}{2}$ and $p_{R}=$ $1-F\left(x_{n / 2}\right)>\frac{1}{2}$. Now candidates would expect to win with larger probability if they chose policies on the right side of the policy space since they believe that the median voter is most likely to be rightist. In this case, by symmetry, we would have that the unique SE in undominated strategies should be symmetric to 
the one described for $F\left(x_{n / 2}\right)>\frac{1}{2}$ : the advantaged candidate assigns increasing amounts of probability to policies that are close to one, and according to a mirror image, the disadvantaged candidate assigns increasing amounts of probability to policies that are close to zero.

Remark 1 When $\rho \leq 1-\min \left\{f\left(x_{1}\right), f\left(x_{n}\right)\right\}$ there may exist SE in dominated strategies. For instance, suppose that $n=10, \rho=0.6, F(3 / 9)=0.4, F(4 / 9)=0.55, F(6 / 9)=0.6 .^{7}$ Notice that in this case we have $\rho=0.6>F\left(x_{n / 2}\right)=0.55>\frac{1}{2}$ and $\min \left\{f\left(x_{1}\right), f\left(x_{n}\right)\right\} \leq 0.4$, thus, $\rho=0.6 \leq 1-\min \left\{f\left(x_{1}\right), f\left(x_{n}\right)\right\}$. So, if candidate $A$ chooses policy $5 / 9$ with probability one and candidate $B$ chooses policies $2 / 9$ and 8/9 each with probability $\frac{1}{2}$, and the voter's beliefs are $b_{L}(5 / 9,8 / 9)=1, b_{I}(5 / 9,5 / 9)=1, b_{R}(5 / 9,2 / 9)=1$ we have a SE in dominated strategies. ${ }^{8}$

Remark 2 When $\rho>1-\min \left\{f\left(x_{1}\right), f\left(x_{n}\right)\right\}$ there are no SE in dominated strategies. That is, the game admits a unique SE (the one described in proposition 3). F having full support means that $f\left(x_{1}\right)>0$ and $f\left(x_{n}\right)>0$. That is, when we are in information set $L$, the voter expects higher utility from $A$ if $x_{m}=x_{1}$ and higher utility from $B$ if $x_{m}=x_{n}$ for any $b_{L}$; therefore $p_{L} \in\left[f\left(x_{1}\right), 1-f\left(x_{n}\right)\right]$. Equivalently, when we are in information set $R$, the voter expects higher utility from $A$ if $x_{m}=x_{n}$ and higher utility from $B$ if $x_{m}=x_{1}$ for any $b_{R}$; therefore $p_{R} \in\left[f\left(x_{n}\right), 1-f\left(x_{1}\right)\right]$. So if $\rho>1-\min \left\{f\left(x_{1}\right), f\left(x_{n}\right)\right\}$ then for any possible $b_{L}$ and $b_{R}$ we have that $\rho>\max \left\{p_{L}, p_{R}\right\}$ (and this should obviously hold in a SE too). And as we know from propositions 1 and 3 there exists a unique $S E$ such that $\rho>\max \left\{p_{L}, p_{R}\right\}$ and it is the one described in proposition $3 .^{9}$

\footnotetext{
${ }^{7}$ Remember that for $n=10$ we have that $x_{k}=\frac{k-1}{9}$.

${ }^{8}$ To see why this is a SE consider the following fully mixed strategies:

$$
\sigma_{6}^{A}=1-\left(\frac{1}{\lambda}\right)^{\lambda}, \sigma_{k}^{A}=\frac{1}{9}\left(\frac{1}{\lambda}\right)^{\lambda} \text { for } k \neq 6
$$

and

$$
\sigma_{3}^{B}=\sigma_{9}^{B}=\frac{1}{2}-\frac{1}{\lambda}, \sigma_{k}^{B}=\frac{1}{8} \frac{2}{\lambda} \text { for } k \notin\{3,9\}
$$

for some $\lambda>2$.

We observe that for $\lambda \rightarrow+\infty$ we have that $\sigma_{6}^{A} \rightarrow 1, \sigma_{3}^{B}=\sigma_{9}^{B} \rightarrow \frac{1}{2}$ and $\frac{\sigma_{6}^{A} \sigma_{6}^{B}}{\sum_{i=1}^{n} \sigma_{i}^{A} \sigma_{i}^{B}} \rightarrow 1$.

Hence these strategies converge to the described strategy profile and they are fully mixed. If the voter has beliefs consistent with candidates playing these strategies it should be the case that $b_{L}\left(x_{6}, x_{9}\right)=b_{R}\left(x_{6}, x_{3}\right) \rightarrow 1$ and $b_{I}\left(x_{6}, x_{6}\right) \rightarrow 1$.

${ }^{9}$ The arguments presented in Remark 2 also prove that when $\rho>1-\min \left\{f\left(x_{1}\right), f\left(x_{n}\right)\right\}$, the unique SE of the game is also the unique Perfect Bayesian Equilibrium of the game. That is, for large values of the advantage the equilibrium prediction of (nearly) maximum differentiation is very robust.
} 
Finally, we deal with the case $F\left(x_{n / 2}\right)=\frac{1}{2}$. First notice that if $\rho=F\left(x_{n / 2}\right)=\frac{1}{2}$ then there is no $\mathrm{SE}$ in undominated strategies. This is expected for the simple reason that in this corner case the game satisfies two notions of symmetry at the same time: both parties are completely identical $\left(\rho=\frac{1}{2}\right)$ and the probability with which the voter is leftist is identical to the probability with which the voter is rightist $\left(F\left(x_{n / 2}\right)=\frac{1}{2}\right)$. Hence, in any SE we have that $p_{L}=\frac{1}{2}=p_{R}$ and, thus, each equilibrium strategy is veryweakly dominated by any other strategy. However, when we break at least one of these symmetries we have existence and uniqueness of a SE in undominated strategies. The case with asymmetric distribution of the median voter's ideal point and identical candidates has already been included in the previous analysis. Here we focus on the case with symmetric distribution of the median voter's ideal point and an advantaged candidate, that is, $\rho>F\left(x_{n / 2}\right)=\frac{1}{2}$. We show that in this case we also have existence and uniqueness of SE in undominated strategies.

Proposition $4 A$ unique $S E$ in undominated strategies exists and it is such that the candidates' strategies are $\sigma_{k}^{* A}=\sigma_{k}^{* B}=\frac{1}{n}$ for $k=1, \ldots, n$, and $b_{L}=b_{R}=\frac{2}{n(n-1)}, b_{I}=\frac{1}{n}$ if and only if $\rho>F\left(x_{n / 2}\right)=\frac{1}{2}$.

When we have that the ideal point of the median voter is equally likely to be rightist or leftist, candidates do not have a special interest in occupying the extremes of the policy space. But the presence of the advantage in a rather symmetric environment induces the disadvantaged candidate to mix in order to improve his chances of winning. In fact, as the bias of the median voter expected location decreases $\left(F\left(x_{n / 2}\right)\right.$ approaches $\left.\frac{1}{2}\right)$ the slope of the equilibrium probabilities for both candidates decreases: they assign less probability to extreme policies and assign increasing amounts of probability to more centrist policies. Hence, $F\left(x_{n / 2}\right)=\frac{1}{2}$ is a limiting case and it is such that the equilibrium strategies for both candidates become uniform distributions over all the policy space.

Combining the results of the previous propositions for $F\left(x_{n / 2}\right)>\frac{1}{2}$ we have a unique SE in undominated strategies for all $\rho \in\left[\frac{1}{2}, 1\right]$. If $\rho \leq F\left(x_{n / 2}\right)$ by proposition 2 we know that in any SE in undominated strategies, the strategies of both candidates concentrate all of their probability in the left extreme of the policy space. If $\rho>F\left(x_{n / 2}\right)$ by proposition 3 we know that in the unique SE in undominated strategies, the strategies of the candidates concentrate most of their probability in different extremes of the policy space. Hence for any pair of $\rho \in\left[\frac{1}{2}, 1\right]$ and $F\left(x_{n / 2}\right)>\frac{1}{2}$ there exists an essentially unique SE in undominated strategies.

Similarly we could show that for $F\left(x_{n / 2}\right)<\frac{1}{2}$ we have an essentially unique SE in undominated strategies for all $\rho \in\left[\frac{1}{2}, 1\right]$. If $\rho<1-F\left(x_{n / 2}\right)$ in any SE in undominated strategies, the strategies of both 
candidates concentrate all of their probability in the right extreme of the policy space. If $\rho>1-F\left(x_{n / 2}\right)$ in the unique SE in undominated strategies, the strategies of the candidates concentrate most of their probability in different extremes of the policy space. Hence for any pair of $\rho \in\left[\frac{1}{2}, 1\right]$ and $F\left(x_{n / 2}\right)<\frac{1}{2}$ there exists an essentially unique SE in undominated strategies. And we have shown also existence and uniqueness of equilibrium for $\rho>F\left(x_{n / 2}\right)=\frac{1}{2}$. Thus we can state the following result:

Corollary 1 A unique $S E$ in undominated strategies exists for any $\rho \in\left[\frac{1}{2}, 1\right]$ and any $F\left(x_{n / 2}\right) \in\left[\frac{1}{2}, 1\right]$ except for $\left(\rho, F\left(x_{n / 2}\right)\right)=\left(\frac{1}{2}, \frac{1}{2}\right)$.

\section{$5 \quad$ Properties of the Equilibria}

We have shown that a SE in undominated strategies exists for almost all parameter values (except for $\left.F\left(x_{n / 2}\right)=\rho=\frac{1}{2}\right)$ and that in all cases the equilibrium strategies are uniquely defined. Furthermore, we have shown that whenever $F\left(x_{n / 2}\right) \neq \frac{1}{2}$ the candidates' equilibrium strategies tend to the extremes of the policy space. It is only when $F\left(x_{n / 2}\right)=\frac{1}{2}$ that the mixed strategies used in equilibrium are uniform over the policy space. In this section we analyze the equilibrium strategies, payoffs and beliefs for all possible parameter values. In addition we show that our equilibrium results are robust to increases of the voters' information about the candidates' platforms.

\subsection{Strategies}

In the pure strategy equilibrium we found that the candidates' strategies converge to policy $x_{1}=0$ when $\frac{1}{2} \leq \rho \leq F\left(x_{n / 2}\right)$, and converge to policy $x_{n}=1$ when $1-F\left(x_{n / 2}\right) \geq \rho \geq \frac{1}{2}$. In this case the equilibrium payoffs of candidate $A$ are given by its probability of winning which is $\rho>\frac{1}{2}$, since in equilibrium both candidates choose the same policy. Notice that this equilibrium relies on the asymmetry of the expectation of the candidates about the voter's ideal point. The further $F\left(x_{n / 2}\right)$ is from $\frac{1}{2}$ the larger the set of values of $\rho$ for which this equilibrium exists, and when $F\left(x_{n / 2}\right)$ approaches $\frac{1}{2}$ this equilibrium ceases to exist. The comparative statics are more complex for the mixed strategy equilibrium.

In the mixed strategy equilibrium, candidate $A$ assigns increasing probability to policies that are close to $x_{1}=0$ and candidate $B$ uses a mirror image strategy, that is, he assigns increasing probability to policies that are close to $x_{n}=1$. The extremism of this equilibrium policies is large for larger values 
of $F\left(x_{n / 2}\right)$, that is, for more asymmetric expectations of the candidates about the voter's ideal point, candidate $A$ assigns more probability to policies that are close to $x_{1}=0$ and decreases the probability that he assigns to policies larger than $\frac{1}{2}$. Observe that

$$
\frac{\partial \sigma_{k}^{* A}}{\partial H^{*}}=\frac{H^{* k-2}\left[k\left(1-H^{*}\right)-1+H^{* n}\left(1+(n-k)\left(1-H^{*}\right)\right)\right]}{\left(1-H^{* n}\right)^{2}}
$$

and since $H^{*}<1$ we have that $\frac{\partial \sigma_{k}^{* A}}{\partial H^{*}}<0$ holds only for small values of $k$, because as $k$ increases $k\left(1-H^{*}\right)-1$ becomes positive and $\frac{\partial \sigma_{k}^{* A}}{\partial H^{*}}$ turns positive for large values of $k$. On the other hand, since $H^{*}=\frac{\rho-F\left(x_{n / 2}\right)}{\rho-1+F\left(x_{n / 2}\right)}$ we have that $\frac{\partial H^{*}}{\partial F\left(x_{n / 2}\right)}=\frac{1-2 \rho}{\left(\rho-1+F\left(x_{n / 2}\right)\right)^{2}}<0$. Thus we have that

$$
\frac{\partial \sigma_{k}^{* A}}{\partial F\left(x_{n / 2}\right)}=\frac{\partial \sigma_{k}^{* A}}{\partial H^{*}} \frac{\partial H^{*}}{\partial F\left(x_{n / 2}\right)}>0
$$

for small $k$ and the opposite holds when $k$ is large enough.

Also observe that for larger values of $n$ the proportion of policies with increasing probability is smaller. This means that for larger values of $F\left(x_{n / 2}\right)$ the probability with which candidate $A$ chooses rightist policies decreases while the probability with which he chooses leftist policies increases. For candidate $B$ we have a symmetric effect: when $F\left(x_{n / 2}\right)$ increases, he assigns less probability to policies that are close to $x_{1}=0$ and increases the probability he assigns to policies that are close to $x_{n}=1$. Because we have that

$$
\frac{\partial \sigma_{k}^{* B}}{\partial H^{*}}=\frac{\left(H^{*}\right)^{n-k-1}\left[n-k-(n-k+1) H^{*}\right]+\left(H^{*}\right)^{2 n-k-1}\left[k-(k-1) H^{*}\right]}{\left(1-\left(H^{*}\right)^{n}\right)^{2}}
$$

and since $H^{*}<1$ we have that $\frac{\partial \sigma_{k}^{* B}}{\partial H^{*}}>0$ holds only for small values of $k$, because as $k$ increases $\left[n-k-(n-k+1) H^{*}\right]$ becomes negative and $\frac{\partial \sigma_{k}^{* B}}{\partial H^{*}}$ turns negative for large values of $k$. Thus we have that

$$
\frac{\partial \sigma_{k}^{* B}}{\partial F\left(x_{n / 2}\right)}=\frac{\partial \sigma_{k}^{* A}}{\partial H^{*}} \frac{\partial H^{*}}{\partial F\left(x_{n / 2}\right)}<0
$$

for small $k$ and the opposite holds when $k$ is large enough.

The candidates' equilibrium strategies also change with the size of the advantage of candidate $A$. In this case we have that when the value of $\rho$ increases, candidate $A$ decreases the probability that he 
assigns to policies that are close to $x_{1}=0$ and increases the probability assigned to policies that are larger than $\frac{1}{2}$, because using the argument described above to compute the sign of $\frac{\partial \sigma_{k}^{* A}}{\partial H^{*}}$ and given that $\frac{\partial H^{*}}{\partial \rho}=\frac{2 F\left(x_{n / 2}\right)-1}{\left(\rho-1+F\left(x_{n / 2}\right)\right)^{2}}>0$, we have that $\frac{\partial \sigma_{k}^{* A}}{\partial \rho}=\frac{\partial \sigma_{k}^{* A}}{\partial H^{*}} \frac{\partial H^{*}}{\partial \rho}<0$ for small $k$ and the opposite holds when $k$ is large enough.

Similarly, for candidate $B$ we have that when $\rho$ increases, he assigns more probability to policies that are close to $x_{1}=0$ and decreases the probability he assigns to policies that are close to $x_{n}=1$, because in this case we have that $\frac{\partial \sigma_{k}^{* A}}{\partial \rho}=\frac{\partial \sigma_{k}^{* A}}{\partial H^{*}} \frac{\partial H^{*}}{\partial \rho}>0$ for small $k$ and the opposite holds when $k$ is large enough.

When the number of policies in the policy space increases we have that the probability assigned to each policy decreases $\left(\frac{\partial \sigma_{k}^{* A}}{\partial n}=\frac{H^{* n+k-1}\left(1-H^{*}\right)}{\left(1-H^{* n}\right)^{2}} \ln H^{*}<0\right.$ and $\left.\frac{\partial \sigma_{k}^{* B}}{\partial n}=\frac{H^{* n-k}\left(1-H^{*}\right)}{\left(1-H^{* n}\right)^{2}} \ln H^{*}<0\right)$. More interestingly, when the number of policies goes to infinity we find that in the limit the equilibrium strategies show maximal extremism and maximal differentiation for any $\rho \in\left[\frac{1}{2}, 1\right]$. Let $\lfloor x\rfloor$ denote the smallest integer of $x \in \Re$.

Proposition 5 For any $a \in(0,1), \lim _{n \rightarrow \infty} \sum_{k=1}^{\lfloor a n\rfloor} \sigma_{k}^{* A}(n)=1$ and $\lim _{n \rightarrow \infty} \sum_{k=\lfloor(1-a) n\rfloor}^{n} \sigma_{k}^{* B}(n)=1$.

In plain words, when we look at the limiting case where the discrete grid on the policy space becomes arbitrarily fine and the advantage becomes arbitrarily small, we find that the distributions of the strategies of both candidates approach two degenerate distributions with all mass in each one of the extreme of the policy space: the advantaged candidate on the extreme most likely preferred by the voter and the disadvantaged candidate on the opposite extreme. Thus in the limit both candidates' strategies show maximal differentiation concentrating their probability on each one of the most extreme policies.

\subsection{Payoffs}

Since we assume that in case of indifference the voter votes for candidate $A$ with probability $\rho \geq \frac{1}{2}$, we expect that the advantage of candidate $A$ will show in the equilibrium results. Notice that the equilibrium payoffs for candidate $A$ can be written as:

$$
\Pi^{A}\left(\sigma^{* A}, \sigma^{* B} \mid b_{L}, b_{I}, b_{R}\right)=\Pi^{A}\left(x_{1}, \sigma^{* B} \mid b_{L}, b_{I}, b_{R}\right)=\rho \sigma_{1}^{* B}+\left(1-\sigma_{1}^{* B}\right) F\left(x_{n / 2}\right)
$$


because in the mixed strategy equilibrium candidate $A$ must be indifferent among all pure strategies in his support, and in particular, the payoff he obtains when he plays $x_{1}$ is as shown above: with probability $\sigma_{1}^{* B}$ both candidates choose the same strategy and candidate $A$ wins with probability $\rho$ and, otherwise, candidate $B$ chooses a strategy to the right of candidate $A$, thus the voter gets signal $L$ and she is expected to vote for candidate $A$ with probability $p_{L}=F\left(x_{n / 2}\right)$. Therefore, candidate $A$ 's payoffs in equilibrium are larger than candidate $B$ 's, because we have that both $\rho$ and $F\left(x_{n / 2}\right)$ are larger than $\frac{1}{2}$. In fact since $\rho>F\left(x_{n / 2}\right) \geq \frac{1}{2}$ we have that $\rho>\Pi^{A}\left(\sigma^{* A}, \sigma^{* B} \mid b_{L}, b_{I}, b_{R}\right)>F\left(x_{n / 2}\right) \geq \frac{1}{2}$. Moreover, they are increasing in $\rho$ because $A$ 's payoffs can also be written as

$$
\Pi^{A}\left(\sigma^{* A}, \sigma^{* B} \mid b_{L}, b_{I}, b_{R}\right)=\Pi^{A}\left(x_{n}, \sigma^{* B} \mid b_{L}, b_{I}, b_{R}\right)=\rho \sigma_{n}^{* B}+\left(1-\sigma_{n}^{* B}\right)\left(1-F\left(x_{n / 2}\right)\right)
$$

and hence

$$
\frac{\partial \Pi^{A}\left(x_{n}, \sigma^{* B} \mid b_{L}, b_{I}, b_{R}\right)}{\partial \rho}=\sigma_{n}^{* B}+\left(\rho-\left(1-F\left(x_{n / 2}\right)\right)\right) \frac{\partial \sigma_{n}^{* B}}{\partial H^{*}} \frac{\partial H^{*}}{\partial \rho}>0
$$

since $\frac{\partial H^{*}}{\partial \rho}>0$ and $\frac{\partial \sigma_{n}^{* B}}{\partial H^{*}}=\frac{H^{* n-2}\left(n\left(1-H^{*}\right)-1+H^{* n}\right)}{\left(1-H^{* n}\right)^{2}}>0$ for every $H^{*}<1$. In figures $3 \mathrm{a}$ and $3 \mathrm{~b}$ we present examples of how candidate $A$ 's equilibrium payoffs relate to the value of the advantage.

[Insert Figures 3a and 3b about here]

Observe that the payoffs of candidate A are always increasing with the size of the advantage. When the advantage size is smaller than the bias of the distribution, the payoffs of candidate A are increasing proportionally to the size of the advantage. However, when the advantage size is larger than the bias of the distribution, the payoffs of candidate A are only weakly increasing, and they become flatter for larger values of $n$.

Furthermore we have that the equilibrium payoffs of candidate $A$ decrease with $n$, because as the number of policies increases the effect of the advantage vanishes. This is so because

$$
\Pi^{A}\left(\sigma^{* A}, \sigma^{* B} \mid b_{L}, b_{I}, b_{R}\right)=F\left(x_{n / 2}\right)+\left(\rho-F\left(x_{n / 2}\right)\right) \frac{H^{* n-1}\left(1-H^{*}\right)}{1-H^{* n}}
$$

implies that 


$$
\frac{\partial \Pi^{A}\left(\sigma^{* A}, \sigma^{* B}\right)}{\partial n}=\left(\rho-F\left(x_{n / 2}\right)\right) \frac{H^{* n-1}\left(1-H^{*}\right) \ln H^{*}}{\left(1-H^{* n}\right)^{2}}<0
$$

We also have that as the number of equidistant policies goes to infinity the payoff of candidate $A$ in equilibrium converges to the probability with which the ideal point of the voter is expected to be on the left side of the policy space.

$$
\lim _{n \rightarrow \infty} \Pi^{A}\left(\sigma^{* A}, \sigma^{* B} \mid b_{L}, b_{I}, b_{R}\right)=F\left(x_{n / 2}\right) .
$$

Finally, observe that as pointed above, the equilibrium payoffs of candidate $A$ are increasing with $F\left(x_{n / 2}\right)$ in all cases:

$$
\frac{\partial \Pi^{A}\left(\sigma^{* A}, \sigma^{* B} \mid b_{L}, b_{I}, b_{R}\right)}{\partial F\left(x_{n / 2}\right)}=\frac{\partial \Pi^{A}\left(\sigma^{* A}, \sigma^{* B} \mid b_{L}, b_{I}, b_{R}\right)}{\partial H^{*}} \frac{\partial H^{*}}{\partial F\left(x_{n / 2}\right)}
$$

and since

$$
\frac{\partial \Pi^{A}\left(\sigma^{* A}, \sigma^{* B} \mid b_{L}, b_{I}, b_{R}\right)}{\partial H^{*}}=\left(\rho-F\left(x_{n / 2}\right)\right) \frac{H^{* n-2}\left(n\left(1-H^{*}\right)-1\right)\left(1-H^{* n}\right)+n H^{* 2(n-1)}\left(1-H^{*}\right)}{\left(1-H^{* n}\right)^{2}}<0
$$

it trivially follows that $\frac{\partial \Pi^{A}\left(\sigma^{* A}, \sigma^{* B} \mid b_{L}, b_{I}, b_{R}\right)}{\partial F\left(x_{n / 2}\right)}>0$.

Since this is a zero sum game, the comparative statics for the payoffs of candidate $B$ can be derived from the previous results.

\section{$5.3 \quad$ Beliefs}

The voter's beliefs in equilibrium after observing any given signal $S$ are given by:

$$
\begin{gathered}
b_{L}\left(x_{i}, x_{i^{\prime}}\right)=\frac{\left(H^{*}\right)^{n-i^{\prime}+i}\left(1-H^{*}\right)^{2}}{H^{*}+\left(H^{*}\right)^{n}\left[(n-1) H^{*}-n\right]} \text { for }\left(x_{i}, x_{i^{\prime}}\right) \in Y_{L}, \\
b_{I}\left(x_{i}, x_{i^{\prime}}\right)=\frac{1}{n} \text { for }\left(x_{i}, x_{i^{\prime}}\right) \in Y_{I}, \\
b_{R}\left(x_{i}, x_{i^{\prime}}\right)=\frac{\left(H^{*}\right)^{-i^{\prime}+i-1}\left(1-H^{*}\right)^{2}}{\left(H^{*}\right)^{n}-1+n\left(1-H^{*}\right)} \text { for }\left(x_{i}, x_{i^{\prime}}\right) \in Y_{R},
\end{gathered}
$$


Notice that for every $S \in\{L, R\}$ we have

$$
\frac{\partial b_{S}\left(x_{i}, x_{i^{\prime}}\right)}{\partial i}=b_{S}\left(x_{i}, x_{i^{\prime}}\right) \ln H^{*}<0
$$

and

$$
\frac{\partial b_{S}\left(x_{i}, x_{i^{\prime}}\right)}{\partial i^{\prime}}=-b_{S}\left(x_{i}, x_{i^{\prime}}\right) \ln H^{*}>0 .
$$

This implies that when the voter observes any signal she rightly assigns larger probability to candidate $A$ choosing policies that are closer to $x_{1}=0$ and larger probability to candidate $B$ choosing policies that are closer to $x_{n}=1$.

Now define $\gamma=\left|i-i^{\prime}\right|$ (the distance between the policy platforms of the two candidates). We see that

$$
\frac{\partial b_{L}\left(x_{i}, x_{i^{\prime}}\right)}{\partial \gamma}=-b_{L}\left(x_{i}, x_{i^{\prime}}\right) \ln H^{*}>0
$$

which suggests that after signal $L$ the voter assigns higher probability to more distant pairs of policy platforms (notice that $b_{L}\left(x_{i}, x_{i^{\prime}}\right)$ is maximized at $(0,1)$ )

and that

$$
\frac{\partial b_{R}\left(x_{i}, x_{i^{\prime}}\right)}{\partial \gamma}=b_{R}\left(x_{i}, x_{i^{\prime}}\right) \ln H^{*}<0
$$

which suggests that after signal $R$ the voter assigns higher probability to more neighboring pairs of policy platforms (notice that $b_{R}\left(x_{i}, x_{i^{\prime}}\right)$ is maximized at $\left(x_{i}, x_{i-1}\right)$ for every $i \in\{2,, 3, \ldots, n\}$ ).

[Insert Figures 4a and 4b about here]

Examples of distributions of the voter's beliefs after signals $L$ and $R$ are illustrated in figures 4 a and 4b. Observe that after signal $L$ the voter assigns larger probability to pairs of distant platforms, that is, she rightly expects that when candidate $A$ is the most leftist, he is expected to choose extreme leftist policies and candidate $B$ is expected to choose extreme rightist policies. While after signal $R$ the voter assigns larger probability to pairs of similar platforms, that is, she rightly expects that when candidate $A$ is the most rightist, both candidates must be choosing neighboring policies. 


\subsection{Informed Voters}

We now consider a generalized model in which the voter actually "sees" the policies that the candidates offer with probability $\varepsilon \in[0,1]$. Otherwise, with probability $1-\varepsilon$, as in the main model, the voter can only observe whether both candidates have chosen the same policy, or when that is not the case, which candidate has chosen a more rightist or leftist policy.

We will argue that for sufficiently small values of $\varepsilon$ this generalized model admits an undominated SE such that the behavior of all players is very similar to the case that we analyzed in the previous sections. That is, we prove that the equilibrium predictions of extremist candidate behavior are robust to the introduction of a small enough probability that the voter is actually informed. Notice that a small probability of our voter being informed corresponds to a tiny fraction of informed voters if we use the interpretation of many voters.

We denote by $\sigma^{*}(\varepsilon)$ the pair of candidates' strategies in an undominated SE of this generalized game. Let $\sigma^{*}(0)$ denote the equilibrium pair of strategies of the extended game corresponding to $\varepsilon=0$ (the equilibrium pair of strategies of the main model). That is, $\sigma^{*}(0)$ denotes the pure strategy profile $\left(x_{1}, x_{1}\right)=(0,0)$ when $\rho \leq F\left(x_{n / 2}\right)$ and it denotes the mixed strategy profile of proposition 3 when $\rho>F\left(x_{n / 2}\right)$.

Proposition 6 For $\rho \neq F\left(x_{n / 2}\right)>\frac{1}{2}$ and $\varepsilon$ sufficiently small there exists an undominated SE such that $\lim _{\varepsilon \rightarrow 0^{+}} \sigma^{*}(\varepsilon)=\sigma^{*}(0)$.

We do not characterize all the SE of the extended game; we only show existence of a sequence of equilibria that approaches the equilibria we found for our main model. Given the characteristics of the sequence, it is normal to conjecture that as $\varepsilon$ increases, the level of extremism exhibited by candidates in equilibrium should decrease ${ }^{10}$. We provide a full characterization of the unique SE for any $\varepsilon \in[0,1]$ for the particular case in which $\rho=1, F$ is uniform and $n=3$. We also show that the degree of extremism, defined as the probability that no candidate offers the moderate platform, in this equilibrium is decreasing in $\varepsilon$.

Proposition 7 If $\rho=1, F$ is uniform and $X=\left\{0, \frac{1}{2}, 1\right\}$ then for any $\varepsilon \in[0,1]$ the unique SE of the game is such that $\sigma_{1}^{* A}(\varepsilon)=\sigma_{3}^{* A}(\varepsilon)=\frac{1}{3+2 \varepsilon}, \sigma_{2}^{* A}(\varepsilon)=\frac{1+2 \varepsilon}{3+2 \varepsilon}$ and $\sigma_{1}^{* B}(\varepsilon)=\sigma_{3}^{* B}(\varepsilon)=\frac{1+\varepsilon}{3+2 \varepsilon}, \sigma_{2}^{* B}(\varepsilon)=\frac{1}{3+2 \varepsilon}$.

\footnotetext{
${ }^{10}$ For the special cases in which either $\rho=F\left(x_{n / 2}\right)$ or $F\left(x_{n / 2}\right)=\frac{1}{2}$ the argument used in the proof does not apply. However we conjecture that continuity would lead to similar results.
} 
Let $\Delta\left(\sigma^{A}, \sigma^{B}\right)$ denote the degree of extremism of strategies $\left(\sigma^{A}, \sigma^{B}\right)$. Observe that in this equilibrium we have that the degree of extremism of the equilibrium strategies is given by

$$
\Delta\left(\sigma^{* A}(\varepsilon), \sigma^{* B}(\varepsilon)\right)=\left(1-\sigma_{2}^{* A}\right)\left(1-\sigma_{2}^{* B}\right)=\frac{4(1+\varepsilon)}{(3+2 \varepsilon)^{2}} .
$$

The degree of extremism of the equilibrium is a function of the proportion of voters that are informed, and since $\frac{\partial \Delta\left(\sigma^{* A}(\varepsilon), \sigma^{* B}(\varepsilon)\right)}{\partial \varepsilon}=-\frac{4(1+2 \varepsilon)}{(3+2 \varepsilon)^{3}}<0$ it is strictly decreasing in $\varepsilon$.

Furthermore, we have that the equilibrium payoffs of candidate $A$ are given by

$$
\Pi^{A *}\left(\sigma^{* A}(\varepsilon), \sigma^{* B}(\varepsilon)\right)=1-\frac{\left(\sigma_{2}^{* A}(\varepsilon)+\sigma_{3}^{* A}(\varepsilon)\right)}{3}=1-\frac{2(1+\varepsilon)}{3(3+2 \varepsilon)}
$$

It is straightforward to see that the payoffs of the advantaged candidate in equilibrium decrease with the proportion of informed voters $\left(\frac{\partial \Pi^{A *}\left(\sigma^{* A}(\varepsilon), \sigma^{* B}(\varepsilon)\right)}{\partial \varepsilon}=-\frac{2}{3(3+2 \varepsilon)^{2}}<0\right)$. Thus, favoring the introduction of voters' information reduces the effect of the advantage and benefits the disadvantaged candidate.

By specifically assuming that $u_{m}(x)=-\left|x-x_{m}\right|$ we can compute the expected social welfare, $W\left(\sigma^{A}, \sigma^{B}\right)$, under a veil of ignorance. That is, if we assume that $x_{m}$ is a random draw from a uniform distribution then, in equilibrium, the voter's expected welfare should be given by

$$
\begin{gathered}
W\left(\sigma^{* A}(\varepsilon), \sigma^{* B}(\varepsilon)\right)=2 \frac{1}{3}\left[\sigma_{2}^{* A}(\varepsilon)\left(1-\sigma_{1}^{* B}(\varepsilon)\right)\left(-\frac{1}{2}\right)+\sigma_{3}^{* A}(\varepsilon) \sigma_{2}^{* B}(\varepsilon)\left(-\frac{1}{2}\right)+\sigma_{3}^{* A}(\varepsilon) \sigma_{3}^{* B}(\varepsilon)(-1)\right]+ \\
+\frac{1}{3}\left[\varepsilon\left(\sigma_{1}^{* A}(\varepsilon)+\sigma_{3}^{* A}(\varepsilon)\right)\left(\sigma_{1}^{* B}(\varepsilon)+\sigma_{3}^{* B}(\varepsilon)\right)\left(-\frac{1}{2}\right)+(1-\varepsilon)\left(\sigma_{1}^{* A}(\varepsilon)+\sigma_{3}^{* A}(\varepsilon)\right)\left(-\frac{1}{2}\right)\right]= \\
=-\frac{2(2+\varepsilon)^{2}}{3(3+2 \varepsilon)^{2}}
\end{gathered}
$$

It is easy to check that expected social welfare increases with the proportion of informed voters $\left(\frac{\partial W\left(\sigma^{* A}(\varepsilon), \sigma^{* B}(\varepsilon)\right)}{\partial \varepsilon}=\frac{4(2+\varepsilon)}{3(3+2 \varepsilon)^{3}}>0\right)$. Hence, as it should be expected, politically informed societies enjoy higher levels of welfare than societies with a small proportion of informed voters.

For any symmetric $F$ the qualitative implications of the above proposition remain intact. That is, a) there exists a unique SE, b) the degree of extremism of this unique equilibrium is strictly decreasing in the probability that the voter is informed and c) expected social welfare is increasing in the probability that the voter is informed. Notice that, when the voter is uninformed with probability one, both candidates 
mix uniformly (this is in line with the general results of the previous section) and when the voter is informed with probability one, the mixed strategies of both candidates coincide with the Aragonès and Palfrey (2002) equilibrium.

\section{Comparison with the conventional model}

In this section, we compare our set of assumptions regarding voters' behavior with voters' preferences in conventional models of electoral competition with an advantaged candidate. The comparison of our model with a generalization of the conventional one will allow us to offer a new interpretation of the model developed here and to provide a rationalization for the assumptions made on the behavior of the voters. To this end we prove that under both sets of assumptions regarding voters' behavior one gets exactly the same results in the imperfect information scenario that is developed in this paper.

In the literature which analyzes electoral competition with an advantaged candidate, a discrete policy space and perfect information regarding candidate's choices (see for example Aragonès and Palfrey 2002) it is assumed that the voter's utility is given by $u_{m}\left(x_{A}\right)=\phi\left(\left|x_{A}-x_{m}\right|\right)+d$ if candidate $A$ is elected and by $u_{m}\left(x_{B}\right)=\phi\left(\left|x_{B}-x_{m}\right|\right)$ if candidate $B$ is elected, where $d>0$ is sufficiently small (so that it plays a role only when the voter is indifferent between the policy locations of the two candidates, that is, when $\left.\phi\left(\left|x_{A}-x_{m}\right|\right)=\phi\left(\left|x_{B}-x_{m}\right|\right)\right)$. Hence, when the voter is indifferent she always votes for the same candidate, the advantaged candidate, instead of randomizing between the two.

We could generalize the conventional model in the following way: the voter's utility is given by $u_{m}\left(x_{A}\right)=\phi\left(\left|x_{A}-x_{m}\right|\right)+\hat{d}$ if candidate $A$ is elected and by $u_{m}\left(x_{B}\right)=\phi\left(\left|x_{B}-x_{m}\right|\right)$ if candidate $B$ is elected, where $\hat{d} \in\{-d, d\}$ for some $d>0$. The two candidates have common but imperfect information regarding the value of $\hat{d}$. They believe that $\hat{d}=d$ with probability $\rho \in\left[\frac{1}{2}, 1\right]$ and that $\hat{d}=-d$ with probability $1-\rho \in\left[0, \frac{1}{2}\right] .{ }^{11}$ As before when $d>0$ is sufficiently small and a voter has perfect information regarding candidate's choices, the advantage plays a role only when the voter is indifferent between the policy locations of the two candidates, that is, when $\phi\left(\left|x_{A}-x_{m}\right|\right)=\phi\left(\left|x_{B}-x_{m}\right|\right)$. In this more general setup when the voter is indifferent between the policy locations of the two candidates she is expected to vote for $A$ with probability $\rho \in\left[\frac{1}{2}, 1\right]$ and for $B$ with probability $1-\rho$.

It is obvious that the voters' preferences of this generalized conventional model resemble very much

\footnotetext{
${ }^{11}$ We can alternatively assume that $\hat{d}$ is continuously distributed in $[d, d]$ according to an arbitrary distribution function $G$. In this case $\rho=1-G(0)$.
} 
(but are not identical to) the assumptions that our model made on the voters' behavior. The main difference lies in the fact that when both candidates offer the same policy then a voter is never indifferent between them; when $\hat{d}=d$ (this occurs with probability $\rho \in\left[\frac{1}{2}, 1\right]$ ) the voter is strictly better off by voting for candidate $A$ and when $\hat{d}=-d$ (this occurs with probability $1-\rho \in\left[0, \frac{1}{2}\right]$ ) the voter is strictly better off by voting for $B$. That is, this generalization of the conventional model offers a solid rational basis for our working assumption regarding the voters' behavior when they are indifferent between the policies that the two candidates propose.

By introducing our informational assumptions (voters do not observe the candidates' policy choices but only which candidate offers the most conservative/liberal policy) in this generalized version of the conventional model, we can actually show that for $d>0$ sufficiently small we obtain the same equilibria with the model that we extensively analyzed in this paper. In the next proposition we prove this for the particular case of $\rho=1$. A generalization to any $\rho \in\left[\frac{1}{2}, 1\right]$ trivially follows from the arguments developed in the proof.

Proposition 8 When $\rho=1$, for any $n$ and $F$ there exists $\hat{d}(n, F)>0$ such that for every $d \in(0, \hat{d}(n, F)]$ the unique SE of a game with the voter's preferences of the conventional game and the imperfect information scenario of our model coincides with the unique SE of our model.

\section{Extensions and concluding remarks}

The previous analysis shows that when we combine a small valence advantage for one of the candidates with imperfect information of the voters about the candidates' platforms we can find a unique equilibrium prediction that depending on the size of the advantage will be in pure or mixed strategies. On the one hand, the advantage induces the candidates to differentiate from each other, and on the other hand, the lack of information of the voters induces the candidates to choose extreme policies. When the advantage is small the candidates' equilibrium choices are mostly driven to the extremes of the policy space, and when the advantage is large the candidates become more moderate. Similarly, we have also shown that when the voters become better informed the candidates' policy choices become less extreme and when the proportion of well informed voters goes to one, the equilibrium policy choices converge to the ones described in Aragonès and Palfrey (2002): the advantaged candidate concentrates most of his probability on moderate policies while the disadvantaged candidate concentrates most of his probability on more extreme policies. 
All these results are proven on a discrete policy space and they hold for any number of equidistant policies. In such a game of imperfect information, a discrete policy space has clear technical advantages when compared to a continuous policy space which we hope that have been made apparent by now. For instance, the construction of sequences of fully mixed strategies which converge to the equilibrium mixed strategies and, hence, the characterization of a sequential equilibrium is distinctly more tractable in finite games than in infinite ones. Moreover, the idea that voters perceive two platforms of being the same not only when they are identical, in the mathematical sense, but also when they are almost identical is easily modeled by the size of the number of distinct policies that are available in the policy space: a large $n$ is equivalent to a voter easily distinguishing which policy is more rightist/leftist and a small value of $n$ captures the case in which a voter can distinguish differences between two policy proposals only if these policies are very far from each other. If we had a continuous policy space instead then, to account for the above idea of not noticeable differences between candidates' platforms, we should develop a more complicated model in which information set $I$ is produced not only when both candidates offer exactly the same policy but also when their distance is smaller than a fixed threshold value. Despite that, we have sufficient formal evidence ${ }^{12}$ which indicates that if we considered instead a continuous policy space we would get similar equilibria with the ones that we find in this finite game (independently of whether the information set $I$ accounts for the idea of not noticeable differences or not) at the cost of a more complicated and impractical analysis (in terms of fully describing equilibrium features and properties).

One can further show that when voters are not only informed about the direction of candidates platforms (as in our model) but also about their distance, then an equilibrium exists in which candidates use the same strategies as in the unique equilibrium of our game for large values of $\rho$. This implies that when one of the candidates enjoys a clear advantage over the other candidate and the number of policy locations is very large, in equilibrium the mixed strategies of the candidates converge to the pure strategy profile where each candidate locates at a different extreme of the policy space and the information of the voter regarding the location of the platform of each candidate is nearly perfect. This is because in this variation of our model the voter gets a signal of the following type "candidate $A$ is more leftist than

\footnotetext{
${ }^{12}$ For example, when the advantage is small relative to the bias of the distribution it is easy to see that all the arguments which support the existence of the described sequential equilibrium (in which both candidates converge to the extreme preferred by the median) directly extend to a model with a continuous policy space (independently of whether $I$ accounts for not noticeable differences or not). When the advantage is large relative to the bias of the distribution the arguments of our proofs do not directly extend to a continuous case but we can still find equilibria that are similar to the unique sequential equilibrium of our finite game. Imagine that $I$ is produced if and only if both candidates announce the same policy platform. Then one can show existence of a sequential equilibrium in which $A$ announces the extreme policy preferred by the median and $B$ 's policy proposal is a random draw from an atomless distribution which includes in its support only policies which are arbitrarily close to the opposite extreme: an equilibrium of (almost) maximal differentiation like the one of our model for an arbitrarily large number of locations.
} 
candidate $B$ and the distance between their platforms is $z^{\prime \prime}$. As we know, when candidates employ the identified mixed strategies and when the policy space is rich, the distance $z$ is with a very large probability very close to one. Hence, the voter will "know" that $A$ chose a policy that is at most $1-z$ away from the left extreme and that candidate $B$ chose a platform that is at most $1-z$ away from the right extreme. That is, extremism and maximum differentiation may arise even in informational setups much richer than the one analyzed in this paper.

\section{References}

[1] Adams, James, Lawrence Ezrow and Zeynep Somer-Topcu. 2011. Is Anybody Listening? Evidence that Voters do not Respond to European Parties' Policy Programmes. American Journal of Political Science, 55 (2), 370-382.

[2] Aragonès, Enriqueta and Thomas Palfrey. 2002. Mixed strategy equilibrium in a Downsian model with a favored candidate. Journal of Economic Theory, 103:131-161.

[3] Aragonès, Enriqueta and Dimitrios Xefteris. 2012. Candidate quality in a Downsian model with a continuous policy space. Games and Economic Behavior, 75:2, 464-480.

[4] Benoit, Kenneth, Slava Mikhaylov, and Michael Laver. 2009. Treating Words as Data with Error: Uncertainty in Text Statements of Policy Positions. American Journal of Political Science, 53 (2), 495-513.

[5] Calvert, Randall L. 1985. Robustness of the Multidimensional Voting Model: Candidate Motivations, Uncertainty and Convergence. Americal Journal of Political Science, 29, 69-95.

[6] Downs, Anthony 1957. An Economic Theory of Democracy. New York, Harper and Row.

[7] Glaeser, Edward L., Giacomo A. M. Ponzetto, and Jesse M. Shapiro. 2005. Strategic extremism: Why Republicans and Democrats divide on religious values. Quarterly Journal of Economics, 120(4):12831330.

[8] Groseclose, Timothy. 2001. A Model of Candidate Location When One Candidate Has a Valence Advantage. American Journal of Political Science, 45 (October): 862-86. 
[9] Grosser, Jens and Thoms Palfrey. 2013. Candidate Entry and Political Polarization: An Antimedian Voter Theorem. American Journal of Political Science, DOI: 10.1111/ajps.12032.

[10] Gul, Faruk and Wolfgang Pesendorfer. 2009. Partisan politics and aggregation failure with ignorant voters. Journal of Economic Theory, 144, 146-174.

[11] Kreps, David M. and Robert B. Wilson. 1982. Sequential equilibria. Econometrica, 50, 863-894.

[12] Laver, Michael, Kenneth Benoit, and John Garry. 2003. Estimating the Policy Positions of Political Actors Using Words as Data. American Political Science Review, 97(2): 311-31.

[13] Laver, Michael, and John Garry. 2000. Estimating Policy Positions from Political Texts. American Journal of Political Science, 44(3), 619-34.

[14] Layman, Geoffrey C., and Thomas M. Carsey. 2002. Party polarization and "conflict extension" in the American electorate. American Journal of Political Science, 46, 786-802.

[15] Lowe, Will, Kenneth Benoit, Mikhaylov, S. and Michael Laver. 2011. Scaling Policy Positions from Hand-Coded Political Texts. Legislative Studies Quarterly, 36 (1), 123-55.

[16] Leslie M. Marx and Jeroen M. Swinkels. 1997. Order independence for iterated weak dominance. Games and Economic Behavior, 18:219-245.

[17] McKelvey, Richard D. and Peter C. Ordeshook. 1985. Elections with Limited Information: A Fulfilled Expectations Model using Contemporaneous Poll and Endorsement Data as Information Sources. Journal of Economic Theory, 36, 55-85.

[18] Mikhaylov, Slava, Michael Laver, and Kenneth Benoit. 2012. Coder Reliability and Misclassification in the Human Coding of Party Manifestos. Political Analysis, 20 (1), 78-91.

[19] Palfrey, Thomas. 1984. Spatial equilibrium with entry. Review of Economic Studies, 51, 139-156.

[20] Rabinowitz, George and Stuart Elaine Macdonald. 1989. A Directional Theory of Voting. American Political Science Review, 83, 93-121.

[21] Roemer, John E. 1994. A Theory of Policy Differentiation in Single Issues Electoral Politics. Social Choice and Welfare, 11, 355-380.

[22] Tomz, Michael and Robert P. Van Houweling. 2008. Positioning and Voter Choice. American Political Science Review, 102(2), 303-18. 
[23] Wittman, Donald. 1977. Candidates with policy preferences: A dynamic model. Journal of Economic Theory, 14, 180-189.

\section{Appendix 1 (Proofs)}

Proof of Proposition 1. a) Since $\rho=p_{I}$, each candidate obtains exactly the same probability of winning, that is, the same payoff, independently of which is the selected pair of strategies. Thus, any strategy is dominated.

b) Consider that in a SE we have $p_{R} \leq \rho \leq p_{L}$ (such that at least one of the two inequalities is strict). Then, we have that 0 is the unique weakly dominant strategy for both candidates. For candidate $A$, for example, any other strategy makes more likely arriving at information set $R$ while not enhancing the probability of arriving at information set $L$. By a similar argument we have that 1 is the unique weakly dominant strategy for both players when $p_{L} \leq \rho \leq p_{R}$. Hence in any SE in undominated strategies in which we have $\rho \in\left[\min \left\{p_{L}, p_{R}\right\}, \max \left\{p_{L}, p_{R}\right\}\right]$, both candidates have to converge to one of the two extremes.

c) Consider that in a $\mathrm{SE}$ we have a belief system $\left(b_{L}, b_{I}, b_{R}\right)$ and assume that there exists a mixed strategy equilibrium $\left(\sigma^{* A}, \sigma^{* B}\right)$ in the reduced game given $\left(b_{L}, b_{I}, b_{R}\right)$ with full support and no gaps $\left(\sigma_{k}^{* A}, \sigma_{k}^{* B}>0\right.$ for all $\left.k\right)$. In the proof we will write $p_{S}$ to denote $p_{S}\left(F, b_{S}\right)$ for $S \in\{L, R, I\}$.

Candidate $A$ knows that if $B$ is playing the mixed strategy that we assume to exist the following will be true: if candidate $A$ selects location $x_{i}$ then with probability $\sum_{k=1}^{i-1} \sigma_{k}^{* B}$ we arrive in information set $R$ and he will get a payoff equal to $p_{R}$, with probability $\sigma_{i}^{* B}$ we arrive in information set $I$ and he will get a payoff equal to $\rho$, and with probability $\sum_{k=i+1}^{n} \sigma_{k}^{* B}$ we arrive in information set $L$ and he will get a payoff equal to $p_{L}$. Thus his expected payoff of selecting $x_{i}$ is:

$$
\Pi^{A}\left(x_{i}, \sigma^{* B}\right)=p_{L} \sum_{k=i+1}^{n} \sigma_{k}^{* B}+\rho \sigma_{i}^{* B}+p_{R} \sum_{k=1}^{i-1} \sigma_{k}^{* B}
$$

and, similarly his expected payoff of selecting $x_{i+1}$ is:

$$
\Pi^{A}\left(x_{i+1}, \sigma^{* B}\right)=p_{L} \sum_{k=i+2}^{n} \sigma_{k}^{* B}+\rho \sigma_{i+1}^{* B}+p_{R} \sum_{k=1}^{i} \sigma_{k}^{* B} .
$$


If $\left(\sigma^{* A}, \sigma^{* B}\right)$ is an equilibrium with full support then we must have that $\Pi^{A}\left(x_{i}, \sigma^{* B}\right)=\Pi^{A}\left(x_{i+1}, \sigma^{* B}\right)$. And this equality implies that:

$$
\frac{\sigma_{i+1}^{* B}}{\sigma_{i}^{* B}}=\frac{\rho-p_{R}}{\rho-p_{L}} \Leftrightarrow \sigma_{i+1}^{* B}=\sigma_{i}^{* B} \frac{\rho-p_{R}}{\rho-p_{L}} \text { for every } i \in\{1,2, \ldots, n-1\}
$$

Let $H=\frac{\rho-p_{L}}{\rho-p_{R}}$. Then, $\sum_{k=1}^{n} \sigma_{k}^{* B}=1$ implies $\sigma_{1}^{* B}+\sigma_{1}^{* B} \frac{1}{H}+\ldots+\sigma_{1}^{* B}\left(\frac{1}{H}\right)^{n-1}=1$ or $\sigma_{1}^{* B}=\frac{1}{\sum_{k=0}^{n-1}\left(\frac{1}{H}\right)^{k}}$ and $\sigma_{i}^{* B}=\sigma_{1}^{* B}\left(\frac{1}{H}\right)^{i-1}$ for $i=2, \ldots, n$.

Notice that for this equilibrium to exist it is necessary that $H>0$, and this implies that in equilibrium we need to have that either $\rho>p_{L}, p_{R}$ or $\rho<p_{L}, p_{R}$. If $H=1$ it trivially follows that $\sigma_{i}^{* B}=\frac{1}{n}$ for all $i \in\{1, \ldots, n\}$. Otherwise, if $H \neq 1$ then $\sigma_{i}^{* B}=H^{n-i} \frac{1-H}{1-H^{n}}$ for $i=1, \ldots, n$.

Using exactly the same steps we can show that $\sigma_{i}^{* A}=\frac{1}{n}$ for all $i \in\{1, \ldots, n\}$ if $H=1$. Otherwise, if $H \neq 1$ then $\sigma_{i}^{* A}=\frac{H^{i-1}(1-H)}{1-H^{n}}$ for $i=1, \ldots, n$.

This shows that there exists a unique full support equilibrium in the reduced game if and only if $H>0$.

Since this reduced game is a constant-sum game, it follows that the set of minimax strategies for both players is convex. If, for example another minimax strategy for player $A$ exists (which should, obviously, be without full support) then any convex combination of the identified minimax strategy and the assumed one should be a minimax strategy as well. But since any convex combination of these two strategies has full support and is different than the identified minimax strategy then infinitely many minimax strategies with full support should exist which contradicts the above finding. Therefore, when this equilibrium exists $(H>0)$ it is unique. Since each player has a unique minimax strategy, it is obviously not dominated, because it could only be dominated by another minimax strategy.

Proof of Proposition 2. By Proposition 1 we have that in a SE in undominated strategies we may have either $p_{L} \neq p_{R}$ and $\rho \in\left[\min \left\{p_{L}, p_{R}\right\}, \max \left\{p_{L}, p_{R}\right\}\right]$ or $\rho>\max \left\{p_{L}, p_{R}\right\}$ or $\rho<\min \left\{p_{L}, p_{R}\right\}$. Let us first argue that for $F\left(x_{n / 2}\right) \geq \rho$ we cannot have a SE in which $\rho>\max \left\{p_{L}, p_{R}\right\}$ or $\rho<\min \left\{p_{L}, p_{R}\right\}$.

Assume that such a SE does exist. Then, as we saw in Proposition 1, and given that $F\left(x_{n / 2}\right)>\frac{1}{2}$ it should be such that $\sigma_{i}^{* A}=\frac{H^{i-1}(1-H)}{1-H^{n}}$ and $\sigma_{i}^{* B}=H^{n-i} \frac{1-H}{1-H^{n}}$ for $i=1, \ldots, n$ and $H=\frac{\rho-p_{L}}{\rho-p_{R}}>0$.

If the voter expects that candidates choose the described strategies, it is obvious, that when we arrive in $I$, that is for $x_{A}=x_{B}$, we have that $E u_{m}\left(\sigma^{* A} \mid I\right)=E u_{m}\left(\sigma^{* B} \mid I\right)$ and therefore the voter votes for $A$ 
with probability $\rho$ and she votes for $B$ with probability $1-\rho$.

If we are in $L$ then we have that:

$E u_{m}\left(\sigma^{* A} \mid L\right)=\sum_{k=1}^{n-1} u_{m}\left(x_{k}\right) \frac{\sigma_{k}^{* A} \sum_{j=k+1}^{n} \sigma_{j}^{* B}}{\sum_{k=1}^{n-1}\left(\sigma_{k}^{* A} \sum_{j=k+1}^{n} \sigma_{j}^{* B}\right)}$

and

$E u_{m}\left(\sigma^{* B} \mid L\right)=\sum_{k=2}^{n} u_{m}\left(x_{k}\right) \frac{\sigma_{k}^{* B} \sum_{j=1}^{k-1} \sigma_{j}^{* A}}{\sum_{k=2}^{n}\left(\sigma_{k}^{* B} \sum_{j=1}^{k-1} \sigma_{j}^{* A}\right)}$.

Notice that since $\sigma_{k}^{* A}=\sigma_{n-k+1}^{* B}$ we have that

$\sum_{k=1}^{n-1}\left(\sigma_{k}^{* A} \sum_{j=k+1}^{n} \sigma_{j}^{* B}\right)=\sum_{k=2}^{n}\left(\sigma_{k}^{* B} \sum_{j=1}^{k-1} \sigma_{j}^{* A}\right)$

and $\sigma_{k}^{* A} \sum_{j=k+1}^{n} \sigma_{j}^{* B}=\sigma_{n-k+1}^{* B} \sum_{j=1}^{n-k} \sigma_{j}^{* A}$.

Furthermore, we know that

$u_{m}\left(x_{k}\right)>u_{m}\left(x_{n-k+1}\right)$ if $x_{m}<\frac{1}{2}$ and $x_{k}<\frac{1}{2}$ or if $x_{m}>\frac{1}{2}$ and $x_{k}>\frac{1}{2}$.

All these imply that:

a) if $x_{m}<\frac{1}{2} \Rightarrow E u_{m}\left(\sigma^{* A} \mid L\right)>E u_{m}\left(\sigma^{* B} \mid L\right)$.

Notice that $E u_{m}\left(\sigma^{* A} \mid L\right)=\sum_{k=1}^{n-1} u_{m}\left(x_{k}\right) \frac{\sigma_{k}^{* A} \sum_{j=k+1}^{n} \sigma_{j}^{* B}}{\sum_{k=1}^{n-1}\left(\sigma_{k}^{* A} \sum_{j=k+1}^{n} \sigma_{j}^{* B}\right)}>\sum_{k=2}^{n} u_{m}\left(x_{k}\right) \frac{\sigma_{k}^{* B} \sum_{j=1}^{k-1} \sigma_{j}^{* A}}{\sum_{k=2}^{n}\left(\sigma_{k}^{* B} \sum_{j=1}^{k-1} \sigma_{j}^{* A}\right)}=E u_{m}\left(\sigma^{* B} \mid L\right)$

iff

$\sum_{k=1}^{n-1} u_{m}\left(x_{k}\right)\left(\sigma_{k}^{* A} \sum_{j=k+1}^{n} \sigma_{j}^{* B}\right)>\sum_{k=2}^{n} u_{m}\left(x_{k}\right)\left(\sigma_{k}^{* B} \sum_{j=1}^{k-1} \sigma_{j}^{* A}\right)$,

because $\sum_{k=1}^{n-1}\left(\sigma_{k}^{* A} \sum_{j=k+1}^{n} \sigma_{j}^{* B}\right)=\sum_{k=2}^{n}\left(\sigma_{k}^{* B} \sum_{j=1}^{k-1} \sigma_{j}^{* A}\right)$

that can be written as

$\left(u_{m}\left(x_{1}\right)-u_{m}\left(x_{n}\right)\right)\left(\sigma_{1}^{* A} \sum_{j=2}^{n} \sigma_{j}^{* B}\right)+\ldots+\left(u_{m}\left(x_{n / 2}\right)-u_{m}\left(x_{n / 2+1}\right)\right)\left(\sigma_{n / 2}^{* A} \sum_{j=n / 2+1}^{n} \sigma_{j}^{* B}\right)+$ 


$$
\begin{aligned}
& +\left(u_{m}\left(x_{n / 2+1}\right)-u_{m}\left(x_{n / 2}\right)\right)\left(\sigma_{n / 2+1}^{* A} \sum_{j=n / 2+2}^{n} \sigma_{j}^{* B}\right)+\ldots+\left(u_{m}\left(x_{n-1}\right)-u_{m}\left(x_{2}\right)\right)\left(\sigma_{n-1}^{* A} \sigma_{n}^{* B}\right)>0, \text { because } \\
& \sigma_{k}^{* A} \sum_{j=k+1}^{n} \sigma_{j}^{* B}=\sigma_{n-k+1}^{* B} \sum_{j=1}^{n-k} \sigma_{j}^{* A}
\end{aligned}
$$

or we can rewrite it as

$$
\begin{aligned}
& \left(u_{m}\left(x_{1}\right)-u_{m}\left(x_{n}\right)\right)\left(\sigma_{1}^{* A} \sum_{j=2}^{n} \sigma_{j}^{* B}\right)+\left(u_{m}\left(x_{2}\right)-u_{m}\left(x_{n-1}\right)\right)\left(\sigma_{2}^{* A} \sum_{j=3}^{n} \sigma_{j}^{* B}-\sigma_{n-1}^{* A} \sigma_{n}^{* B}\right)+\ldots \\
& +\left(u_{m}\left(x_{n / 2}\right)-u_{m}\left(x_{n / 2+1}\right)\right)\left(\sigma_{n / 2}^{* A} \sum_{j=n / 2}^{n} \sigma_{j}^{* B}-\sigma_{n / 2+1}^{* A} \sum_{j=n / 2+2}^{n} \sigma_{j}^{* B}\right)>0,
\end{aligned}
$$

which always holds because $u_{m}\left(x_{k}\right)>u_{m}\left(x_{n-k+1}\right)$ for $k<\frac{1}{2}$ and $x_{m}<\frac{1}{2}$ and

$\sigma_{k}^{* A} \sum_{j=k+1}^{n} \sigma_{j}^{* B}>\sigma_{n-k+1}^{* A} \sum_{j=n-k+2}^{n} \sigma_{j}^{* B}$ also holds because:

$$
\begin{aligned}
& \sigma_{i}^{* A}=\frac{H^{i-1}(1-H)}{1-H^{n}} \text { and } \sigma_{i}^{* B}=\frac{\left(\frac{1}{H}\right)^{i-1}\left(1-\frac{1}{H}\right)}{1-\left(\frac{1}{H}\right)^{n}}=\frac{H^{-i}(H-1)}{1-\frac{1}{H^{n}}}=\frac{H^{n-i}(1-H)}{1-H^{n}} \\
& \sigma_{k}^{* A} \sum_{j=k+1}^{n} \sigma_{j}^{* B}=\frac{H^{k-1}(1-H)}{1-H^{n}} \sum_{j=k+1}^{n} \frac{H^{n-j}(1-H)}{1-H^{n}}=\frac{H^{k-1}(1-H)^{2}}{\left(1-H^{n}\right)^{2}} \sum_{j=0}^{n-k-1} H^{n-j}=\frac{H^{k-1}(1-H)}{\left(1-H^{n}\right)^{2}}\left(1-H^{n-k}\right)
\end{aligned}
$$

and

$$
\begin{aligned}
& \frac{\partial\left(\sigma_{k}^{* A} \sum_{j=k+1}^{n} \sigma_{j}^{* B}\right)}{\partial k}=\frac{\partial\left(\frac{(1-H)}{\left(1-H^{n}\right)^{2}} H^{k-1}\left(1-H^{n-k}\right)\right)}{\partial k}=\frac{(1-H)}{\left(1-H^{n}\right)^{2}}(\ln H)\left(H^{k-1}\right)<0 \text { when } p_{L} \neq p_{R} \\
& \text { while } \frac{\partial\left(\sigma_{k}^{* A} \sum_{j=k+1}^{n} \sigma_{j}^{* B}\right)}{\partial k}=\frac{\partial\left(\frac{n-k}{n^{2}}\right)}{\partial k}<0
\end{aligned}
$$

b) if $x_{m}>\frac{1}{2} \Rightarrow E u_{m}\left(\sigma^{* A} \mid L\right)<E u_{m}\left(\sigma^{* B} \mid L\right), x_{m}<\frac{1}{2} \Rightarrow E u_{m}\left(\sigma^{* A} \mid R\right)<E u_{m}\left(\sigma^{* B} \mid R\right)$ and $x_{m}>\frac{1}{2} \Rightarrow$ $E u_{m}\left(\sigma^{* A} \mid R\right)<E u_{m}\left(\sigma^{* B} \mid R\right)$.

The proof is analogous to the one above.

Therefore, if $x_{m}<\frac{1}{2}$ and we are in $L$ the voter votes for $A$. Notice that $x_{m}<\frac{1}{2}$ is expected to occur with probability $F\left(x_{n / 2}\right)$. If $x_{m}>\frac{1}{2}$ and we are in $L$ the voter votes for $B$. Again, notice that $x_{m}>\frac{1}{2}$ is expected to occur with probability $1-F\left(x_{n / 2}\right)$. The opposite holds when we are in information set $R$.

That is, if candidates choose the described mixed strategies and the voter expects them to do so, the voter is expected to vote for $A$ with probability $\rho$ when we are in $I$; the voter is expected to vote for $A$ with probability $F\left(x_{n / 2}\right)$ when we are in $L$, and the voter is expected to vote for $A$ with probability 
$1-F\left(x_{n / 2}\right)$ when we are in $R$. Notice that this is true for any mixed strategies as the ones described above, that is, for any values of $F$ and $b_{S}$.

Going back to the reduced candidate game we conclude that the only reasonable value for $p_{L}$ is $F\left(x_{n / 2}\right)$ and the only reasonable value for $p_{R}$ is $1-F\left(x_{n / 2}\right)$. If $p_{L}=F\left(x_{n / 2}\right)$ and $p_{R}=1-F\left(x_{n / 2}\right)$, and $F\left(x_{n / 2}\right) \geq \rho$ then it must be the case that $p_{L}=F\left(x_{n / 2}\right) \geq \rho \geq p_{R}=1-F\left(x_{n / 2}\right)$, which implies that $H=\frac{\rho-F\left(x_{n / 2}\right)}{\rho-1+F\left(x_{n / 2}\right)}<0$. Thus if $F\left(x_{n / 2}\right) \geq \rho$ the mixed strategy equilibrium does not exist, and the only possibility for SE in undominated strategies left is the equilibrium in which candidates converge to an extreme of the policy space that exists only for $p_{L} \neq p_{R}$ and $\rho \in\left[\min \left\{p_{L}, p_{R}\right\}, \max \left\{p_{L}, p_{R}\right\}\right]$. In this case, when $F\left(x_{n / 2}\right)>\frac{1}{2}$, we notice that we cannot have a SE in which candidates converge to the right extreme. If there is such a SE there should be a sequence of belief systems such that $\sum_{x \in X} b_{L}(x, 1) \rightarrow 1$. But $F\left(x_{n / 2}\right)>\frac{1}{2}$ along with these beliefs imply that $p_{L}>p_{R}$ and hence we should have $p_{R} \leq \rho \leq p_{L}$ (such that at least one of the two inequalities is strict). Therefore, when $F\left(x_{n / 2}\right)>\frac{1}{2}$ we cannot have $p_{L} \leq \rho \leq p_{R}$ in any $\mathrm{SE}$ in undominated strategies.

This means that the only possibility of existence of an undominated SE when $F\left(x_{n / 2}\right) \geq \rho$ is if it is such that $p_{L} \geq \rho \geq p_{R}$ (with one of the two inequalities being strict). We have shown (proposition 1) that if $p_{L} \geq \rho \geq p_{R}$ (with one of the two inequalities being strict) holds then in an undominated SE both candidates converge to the left extreme. To show that such an undominated SE exists consider the following fully mixed strategies:

$$
\sigma_{1}^{A}=\sigma_{1}^{B}=1-\frac{1}{\lambda}, \sigma_{n}^{A}=\sigma_{n}^{B}=\frac{1}{\lambda}-\left(\frac{1}{\lambda}\right)^{\lambda}
$$

and

$$
\sigma_{k}^{A}=\sigma_{k}^{B}=\frac{1}{n-2}\left(\frac{1}{\lambda}\right)^{\lambda}
$$

for $k \notin\{1, n\}$ and for some $\lambda>2$.

We observe that for $\lambda \rightarrow+\infty$ we have that $\sigma_{1}^{A} \rightarrow 1, \frac{\sigma_{1}^{A}}{\sigma_{k}^{A}} \rightarrow+\infty$ for every $k$ different to 1 and (most importantly) $\frac{\sigma_{n}^{A}}{\sigma_{k}^{A}} \rightarrow+\infty$ for every $k \notin\{1, n\}$.

Hence this strategy converges to the pure strategy 0 when $\lambda \rightarrow+\infty$ and it is a fully mixed strategy. If the voter has beliefs consistent with both players playing this strategy it should be the case that $b_{L}(0,1)=b_{R}(1,0) \rightarrow 1$. This is because $\frac{\sigma_{n}^{A}}{\sigma_{k}^{A}} \rightarrow+\infty$ implies that when $A$ offers a more rightist policy than $B$ it is infinitely more probable that they offer $(1,0)$ compared to any other policy pair. Since 
$F\left(x_{n / 2}\right)>\frac{1}{2}$ such beliefs suggest that $p_{L}=F\left(x_{n / 2}\right)>p_{R}=1-F\left(x_{n / 2}\right)$ and, hence, an undominated SE in which both candidates locate to the left extreme exists if $F\left(x_{n / 2}\right) \geq \rho$. Notice that since $p_{L} \geq \rho \geq p_{R}$ (with one of the two inequalities being strict) implies that both candidates must choose the same pure strategy (the left extreme) in an undominated SE, it follows that in any undominated SE $p_{L} \leq F\left(x_{n / 2}\right)$ and $p_{R} \geq 1-F\left(x_{n / 2}\right)$. Hence, the combination of the conditions $p_{L} \geq \rho \geq p_{R}$ and $p_{L} \leq F\left(x_{n / 2}\right)$ suggests that such a left-convergent undominated SE only if $F\left(x_{n / 2}\right) \geq \rho$.

Proof of Proposition 3. In the end of the proof of Proposition 2 we argued that when $F\left(x_{n / 2}\right)>\frac{1}{2}$ we may have $\rho \in\left[\min \left\{p_{L}, p_{R}\right\}, \max \left\{p_{L}, p_{R}\right\}\right]$ in a $\mathrm{SE}$ in undominated strategies only if $\rho \leq F\left(x_{n / 2}\right)$. So for the case $F\left(x_{n / 2}\right)<\rho$ if a SE in undominated strategies exists it should be such that $\rho>\max \left\{p_{L}, p_{R}\right\}$ or $\rho<\min \left\{p_{L}, p_{R}\right\}$. Proposition 1 indicates that when $\rho>\max \left\{p_{L}, p_{R}\right\}$ or $\rho<\min \left\{p_{L}, p_{R}\right\}$ candidates play $\sigma_{i}^{* A}=\frac{H^{i-1}(1-H)}{1-H^{n}}$ and $\sigma_{i}^{* B}=H^{n-i} \frac{1-H}{1-H^{n}}$ for $i=1, \ldots, n$ and $H=\frac{\rho-p_{L}}{\rho-p_{R}}>0$.

In the proof of Proposition 2 we have shown that if candidates play these fully mixed strategies in a SE, it must be the case that voters' beliefs are consistent with these strategies in every information set and, hence, that the only reasonable value for $p_{L}$ is $F\left(x_{n / 2}\right)$ and the only reasonable value for $p_{R}$ is $1-F\left(x_{n / 2}\right)$. That is, the case $\rho<\min \left\{p_{L}, p_{R}\right\}$ is trivially ruled out and for $F\left(x_{n / 2}\right)<\rho$ we have a unique $\mathrm{SE}$ in undominated strategies such that

$$
\sigma_{k}^{* A}=\left(H^{*}\right)^{k-1} \frac{\left(1-H^{*}\right)}{1-\left(H^{*}\right)^{n}} \text { and } \sigma_{k}^{* B}=\left(H^{*}\right)^{n-k} \frac{\left(1-H^{*}\right)}{1-\left(H^{*}\right)^{n}} \text { for } k=1, \ldots, n \text { with } H^{*}=\frac{\rho-F\left(x_{n / 2}\right)}{\rho-1+F\left(x_{n / 2}\right)}<1 \text {. }
$$

Since this SE involves fully mixed strategies, then every information set is reached with positive probability and, thus, the beliefs of the voter must be as follows:

$$
\begin{aligned}
& b_{L}\left(x_{i}, x_{i^{\prime}}\right)=\frac{\sigma_{i}^{* A} \sigma_{i^{\prime}}^{* B}}{\sum_{k=1}^{n-1}\left[\sigma_{k}^{* A} \sum_{j=k+1}^{n} \sigma_{j}^{* B}\right]} \text { for }\left(x_{i}, x_{i^{\prime}}\right) \in Y_{L}, \\
& b_{I}\left(x_{i}, x_{i^{\prime}}\right)=\frac{\sigma_{i}^{* A} \sigma_{i^{\prime}}^{* B}}{\sum_{k=1}^{n} \sigma_{k}^{* A} \sigma_{k}^{* B}} \text { for }\left(x_{i}, x_{i^{\prime}}\right) \in Y_{I} \\
& b_{R}\left(x_{i}, x_{i^{\prime}}\right)=\frac{\sigma_{i}^{* A} \sigma_{i^{\prime}}^{* B}}{\sum_{k=1}^{n-1}\left[\sigma_{k}^{* B} \sum_{j=k+1}^{n} \sigma_{j}^{* A}\right]} \text { for }\left(x_{i}, x_{i^{\prime}}\right) \in Y_{R} .
\end{aligned}
$$

Notice that since $\sigma_{i}^{* A}=\frac{\left(H^{*}\right)^{i-1}\left(1-H^{*}\right)}{1-\left(H^{*}\right)^{n}}$ and $\sigma_{i}^{* B}=\frac{\left(H^{*}\right)^{n-i}\left(1-H^{*}\right)}{1-\left(H^{*}\right)^{n}}$ we have

$$
\begin{aligned}
& b_{L}\left(x_{i}, x_{i^{\prime}}\right)=\frac{\left(H^{*}\right)^{n+i-i^{\prime}-1}\left(1-H^{*}\right)^{2}}{1-n\left(H^{*}\right)^{n-1}} \text { for }\left(x_{i}, x_{i^{\prime}}\right) \in Y_{L}, \\
& b_{I}\left(x_{i}, x_{i^{\prime}}\right)=\frac{\left(H^{*}\right)^{i-i^{\prime}}}{n} \text { for }\left(x_{i}, x_{i^{\prime}}\right) \in Y_{I}
\end{aligned}
$$




$$
b_{R}\left(x_{i}, x_{i^{\prime}}\right)=\frac{\left(H^{*}\right)^{i-i^{\prime}-1}\left(1-H^{*}\right)^{2}}{n-1-H^{*}+\left(H^{*}\right)^{n}} \text { for }\left(x_{i}, x_{i^{\prime}}\right) \in Y_{R}
$$

Proof of Proposition 4. As in Proposition 1, it is easy to see that we cannot have a SE in undominated strategies for $\rho=p_{L}=p_{R}$.

It is also easy to see that when $F\left(x_{n / 2}\right)=\frac{1}{2}$ we cannot have $\rho \in\left[\min \left\{p_{L}, p_{R}\right\}, \max \left\{p_{L}, p_{R}\right\}\right]$ in a SE in undominated strategies. In the proof of Proposition 2 we have argued that if $p_{L} \geq \rho \geq p_{R}$ holds in a SE in undominated strategies then it should also be the case that $p_{L} \leq F\left(x_{n / 2}\right)$ and that $p_{R} \geq 1-F\left(x_{n / 2}\right)$. When $F\left(x_{n / 2}\right)=\frac{1}{2}$, all these conditions hold if and only if $\rho=p_{L}=p_{R}=\frac{1}{2}$ and, hence, by the first argument of this proof if such a SE exists it cannot be in undominated strategies.

That is, if a $\mathrm{SE}$ in undominated strategies exists for the $F\left(x_{n / 2}\right)=\frac{1}{2}$ case it must be such that $\rho>\max \left\{p_{L}, p_{R}\right\}$ or $\rho<\max \left\{p_{L}, p_{R}\right\}$. We can replicate the arguments in the proof of proposition 1,2 and 3 and assuming that $F\left(x_{n / 2}\right)=\frac{1}{2}$ we obtain that $H^{*}=\frac{\rho-F\left(x_{n / 2}\right)}{\rho-F\left(x_{n / 2}\right)}=1$ thus, we have that $\sigma_{i}^{* A}=\frac{1}{n}$ for $i=1, \ldots, n$ and $p_{L}=\frac{1}{2}=p_{R}$, Thus, as before, in equilibrium we must have that $\rho>\max \left\{p_{L}, p_{R}\right\}$.

Since this SE is fully mixed, then every information set is reached with positive probability and the beliefs of the voter must be as follows:

$$
\begin{aligned}
& b_{L}\left(x_{i}, x_{i^{\prime}}\right)=\frac{\sigma_{i}^{* A} \sigma_{i^{\prime}}^{* B}}{\sum_{k=1}^{n-1}\left[\sigma_{k}^{* A} \sum_{j=k+1}^{n} \sigma_{j}^{* B}\right]}=\frac{2}{n(n-1)} \text { for }\left(x_{i}, x_{i^{\prime}}\right) \in Y_{L}, \\
& b_{I}\left(x_{i}, x_{i^{\prime}}\right)=\frac{\sigma_{i}^{* A} \sigma_{i^{\prime}}^{* B}}{\sum_{k=1}^{n} \sigma_{k}^{* A} \sigma_{k}^{* B}}=\frac{1}{n} \text { for }\left(x_{i}, x_{i^{\prime}}\right) \in Y_{I} \\
& b_{R}\left(x_{i}, x_{i^{\prime}}\right)=\frac{\sigma_{i}^{* A} \sigma_{i^{\prime}}^{* B}}{\sum_{k=1}^{n-1}\left[\sigma_{k}^{* B} \sum_{j=k+1}^{n} \sigma_{j}^{* A}\right]}=\frac{2}{n(n-1)} \text { for }\left(x_{i}, x_{i^{\prime}}\right) \in Y_{R} .
\end{aligned}
$$

Proof of Proposition 5. Since $a \in(0,1)$ we have that:

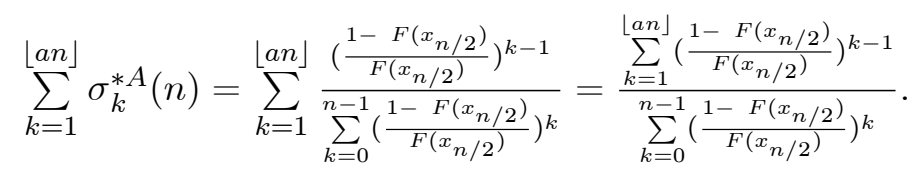

When $n \rightarrow \infty$ we have that both $\lim _{n \rightarrow \infty} \sum_{k=1}^{\lfloor\text {an }}\left(\frac{1-F\left(x_{n / 2}\right)}{F\left(x_{n / 2}\right)}\right)^{k-1}=\frac{1-F\left(x_{n / 2}\right)}{2 F\left(x_{n / 2}\right)-1}$ and $\lim _{n \rightarrow \infty} \sum_{k=0}^{n-1}\left(\frac{1-F\left(x_{n / 2}\right)}{F\left(x_{n / 2}\right)}\right)^{k}=$ $\frac{1-F\left(x_{n / 2}\right)}{2 F\left(x_{n / 2}\right)-1}$ for any $a \in(0,1)$. Therefore, $\sum_{k=1}^{\lfloor a n\rfloor} \sigma_{k}^{* A}(n) \rightarrow 1$ and due to symmetry of $\sigma^{* A}(n)$ and $\sigma^{* B}(n)$ we have $\sum_{k=\lfloor(1-a) n\rfloor}^{n} \sigma_{k}^{* A}(n) \rightarrow 1$ for any $a \in(0,1)$. This implies that we arrive in information set $L$ with 
a probability that converges to one. Moreover we know that if we are in $L$ the voter votes for $A$ with probability $p_{L}=F\left(x_{n / 2}\right)$. Therefore, when $n \rightarrow \infty, A$ is elected with a probability which converges to $F\left(x_{n / 2}\right)>\frac{1}{2}$ and equivalently $B$ is elected with a probability which converges to $1-F\left(x_{n / 2}\right)<\frac{1}{2}$.

Proof of proposition 6. We assume $F\left(x_{n / 2}\right)>\frac{1}{2}$ and consider two cases: $\rho<F\left(x_{n / 2}\right)$ and $\rho>F\left(x_{n / 2}\right)$.

Case $1\left(\rho<F\left(x_{n / 2}\right)\right)$ Consider that voter's beliefs are given by $b_{I}\left(x_{1}, x_{1}\right)=b_{L}\left(x_{1}, x_{n}\right)=b_{R}\left(x_{n}, x_{1}\right)=$ 1 whenever she is uninformed. We have seen in the proof of Proposition 2 why such beliefs can be part of a SE. If this is the case then $\Pi_{A}\left(x_{1}, x_{1}\right)=\rho$ and $\Pi_{A}\left(x_{k}, x_{1}\right) \leq(1-\varepsilon)\left(1-F\left(x_{n / 2}\right)\right)+\varepsilon$ for any $k \neq 1$. Since $\rho<F\left(x_{n / 2}\right)$ and $F\left(x_{n / 2}\right)>\frac{1}{2}$ it trivially follows that $\Pi_{A}\left(x_{k}, x_{1}\right)<\Pi_{A}\left(x_{1}, x_{1}\right)=\rho$ for any $k \neq 1$ and for sufficiently small values of $\varepsilon$. Moreover, $\Pi_{B}\left(x_{1}, x_{1}\right)=1-\rho$ and $\Pi_{B}\left(x_{k}, x_{1}\right) \leq(1-\varepsilon)\left(1-F\left(x_{n / 2}\right)\right)+\varepsilon$ for any $k \neq 1$. Since $\rho<F\left(x_{n / 2}\right) \Longleftrightarrow 1-\rho>1-F\left(x_{n / 2}\right)$ it trivially follows that $\Pi_{B}\left(x_{k}, x_{1}\right)<\Pi_{B}\left(x_{1}, x_{1}\right)=1-\rho$ for any $k \neq 1$ and for sufficiently small values of $\varepsilon$. Hence, for sufficiently small values of $\varepsilon$ there exists an undominated SE in which $\sigma^{*}(\varepsilon)$ is such that $\sigma_{1}^{* A}(\varepsilon)=\sigma_{1}^{* B}(\varepsilon)=1$ and voter's beliefs are $b_{I}\left(x_{1}, x_{1}\right)=b_{L}\left(x_{1}, x_{n}\right)=b_{R}\left(x_{n}, x_{1}\right)=1$.

Case $2\left(\rho>F\left(x_{n / 2}\right)\right)$ Assume that the voter is expected to behave as in the unique equilibrium of the $\varepsilon=0$ case whenever she is uninformed. That is, she votes for the most leftist candidate when $x_{m}<\frac{1}{2}$, for the most rightist candidate when $x_{m}>\frac{1}{2}$ and, whenever we are in information set $I$ she votes for $A$ with probability $\rho$. We will show that this is consistent with what follows at the end of this proof. Obviously, when the voter is informed she votes for the candidate who offers the policy platform nearer to her ideal policy and votes for $A$ with probability $\rho$ whenever she is indifferent between the platforms of both candidates.

Let $\sigma^{*}(\varepsilon)=\left\{\sigma^{* A}(\varepsilon), \sigma^{* B}(\varepsilon)\right\}$ be an equilibrium pair of strategies of the reduced game given the above assumptions about voter's behavior. Observe that the reduced game is a finite constant-sum game and it is, thus, guaranteed to have an equilibrium and a unique equilibrium payoff vector for any value of $\varepsilon$. Moreover, it should be the case that every equilibrium strategy is a minimaximizer strategy as well. First we will argue that if $\sigma^{*}(\varepsilon)$ is an equilibrium of this reduced game then it should be that both candidates use fully mixed strategies for $\varepsilon$ sufficiently small, then we will show that any equilibrium strategy profile of this reduced game should be very close to the unique equilibrium strategy profile of the $\varepsilon=0$ case for $\varepsilon$ sufficiently small and, finally, we will prove that the assumed voter's behavior is consistent with any equilibrium of this reduced game when $\varepsilon$ is sufficiently small establishing the existence of an undominated SE which converges to the undominated SE of the standard model when $\varepsilon \rightarrow 0^{+}$. 
Step 1 Any minimaximizer strategies $\sigma^{* A}(\varepsilon)$ and $\sigma^{* B}(\varepsilon)$ have full support for $\varepsilon$ sufficiently small.

Assume that there exists a minimaximizer strategy for candidate $A, \sigma^{* A}(\varepsilon)$, such that $A$ puts no weight to location $x_{j} \in X\left(\sigma_{j}^{* A}(\varepsilon)=0\right)$. In this case, the payoff of $B$ when $A$ plays $\sigma^{* A}(\varepsilon)$ and $B$ plays $x_{j} \in X, \Pi_{B}\left(\sigma^{* A}(\varepsilon), x_{j}\right)$, should be at most as high as $\Pi_{B}\left(\sigma^{* A}(\varepsilon), \sigma^{* B}(\varepsilon)\right.$ ) (due to the constant-sum nature of the reduced game). Hence, $\Pi_{B}\left(\sigma^{* A}(\varepsilon), \sigma^{* B}(\varepsilon)\right) \geq \Pi_{B}\left(\sigma^{* A}(\varepsilon), x_{j}\right) \geq(1-\varepsilon)\left(1-F\left(x_{n / 2}\right)\right)$ which implies that $\lim _{\varepsilon \rightarrow 0^{+}} \Pi_{B}\left(\sigma^{* A}(\varepsilon), \sigma^{* B}(\varepsilon)\right) \geq 1-F\left(x_{n / 2}\right)$. But we know that when $\rho>F\left(x_{n / 2}\right)$ it is true that $\lim _{\varepsilon \rightarrow 0^{+}} \Pi_{B}\left(\sigma^{* A}(0), \sigma^{B}\right)<1-F\left(x_{n / 2}\right)$ for any possible $\sigma^{B}$. That is, for sufficiently small values of $\varepsilon$, $\sigma^{* A}(\varepsilon)$ cannot be a minimaximizer for $A$ because it gives a payoff to $B$ which converges to something at least as high as $1-F\left(x_{n / 2}\right)$ while if $A$ used $\sigma^{* A}(0)$ then, as $\varepsilon$ approaches zero, the payoff of $B$ would converge to something strictly less than $1-F\left(x_{n / 2}\right)$. So, for $\varepsilon$ sufficiently small, any minimaximizer strategy of candidate $A, \sigma^{* A}(\varepsilon)$, should have full support.

Now, assume that there exists a minimaximizer strategy for candidate $B, \sigma^{* B}(\varepsilon)$, such that $B$ puts no weight to location $x_{1} \in X\left(\sigma_{1}^{* B}(\varepsilon)=0\right)$. Then, since $\rho>F\left(x_{n / 2}\right)$ it is obvious that $\Pi_{A}\left(x_{1}, \sigma^{* B}(\varepsilon)\right)<$ $\Pi_{A}\left(x_{2}, \sigma^{* B}(\varepsilon)\right)$ for any $\varepsilon$. That is, $A$ puts no weight to location $x_{1} \in X$ too; this contradicts the above. So $B$ puts positive weight to location $x_{1}$. Now consider that $\sigma^{* B}(\varepsilon)$ is such that $B$ puts no weight to location $x_{j} \in X\left(\sigma_{j}^{* B}(\varepsilon)=0\right)$ for some $j>1$.

Then:

$$
\Pi_{A}\left(x_{j}, \sigma^{* B}(\varepsilon)\right) \leq(1-\varepsilon)\left[\left(1-F\left(x_{n / 2}\right)\right) \sum_{k=1}^{j-1} \sigma_{k}^{* B}(\varepsilon)+F\left(x_{n / 2}\right) \sum_{k=j+1}^{n} \sigma_{k}^{* B}(\varepsilon)\right]+\varepsilon
$$

That is, for $\varepsilon$ sufficiently small, $\Pi_{A}\left(x_{j}, \sigma^{* B}(\varepsilon)\right)<F\left(x_{n / 2}\right)$. But we know that $\Pi_{A}\left(\sigma^{* A}(0), \sigma^{* B}(\varepsilon)\right)$ converges to something strictly larger than $F\left(x_{n / 2}\right)$ when $\varepsilon$ approaches zero. So $A$ puts no weight to location $x_{j} \in X$ too; this contradicts what we found above. That is, for $\varepsilon$ sufficiently small, any minimaximizer strategies $\sigma^{* A}(\varepsilon)$ and $\sigma^{* B}(\varepsilon)$ should have full support.

Step $\mathscr{2} \sigma^{* A}(\varepsilon) \rightarrow \sigma^{* A}(0)$ and $\sigma^{* B}(\varepsilon) \rightarrow \sigma^{* B}(0)$ when $\varepsilon \rightarrow 0^{+}$.

Since this reduced game is constant sum it must be the case that $\Pi_{A}\left(\sigma^{* A}(\varepsilon), \sigma^{* B}(\varepsilon)\right) \rightarrow \Pi_{A}\left(\sigma^{* A}(0), \sigma^{* B}(0)\right)$ (and $\Pi_{B}\left(\sigma^{* A}(\varepsilon), \sigma^{* B}(\varepsilon)\right) \rightarrow \Pi_{B}\left(\sigma^{* A}(0), \sigma^{* B}(0)\right)$ ) when $\varepsilon \rightarrow 0^{+}$. To see why this holds, assume for example that $\lim _{\varepsilon \rightarrow 0^{+}} \Pi_{A}\left(\sigma^{* A}(\varepsilon), \sigma^{* B}(\varepsilon)\right)>\Pi_{A}\left(\sigma^{* A}(0), \sigma^{* B}(0)\right)\left(\lim _{\varepsilon \rightarrow 0^{+}} \Pi_{B}\left(\sigma^{* A}(\varepsilon), \sigma^{* B}(\varepsilon)\right)<\Pi_{B}\left(\sigma^{* A}(0), \sigma^{* B}(0)\right)\right)$. Then if $B$ uses $\sigma^{* B}(0)$ he should get a payoff which converges to something at least as high as $\Pi_{B}\left(\sigma^{* A}(0), \sigma^{* B}(0)\right)$ when $\varepsilon \rightarrow 0^{+} ; \sigma^{* B}(\varepsilon)$ cannot be a minimaximizer strategy and, thus, part of an equilibrium. 
We proceed now to the main argument. Assume that there exists a minimaximizer strategy for candidate $A, \sigma^{* A}(\varepsilon)$, such that $\lim _{\varepsilon \rightarrow 0^{+}} \sigma_{1}^{* A}(\varepsilon)>\sigma_{1}^{* A}(0)$ (the proof for the $\lim _{\varepsilon \rightarrow 0^{+}} \sigma_{1}^{* A}(\varepsilon)<\sigma_{1}^{* A}(0)$ case is symmetric). Then, since any minimaximizer strategies $\sigma^{* A}(\varepsilon)$ and $\sigma^{* B}(\varepsilon)$ have full support for $\varepsilon$ sufficiently small it must be the case that $\lim _{\varepsilon \rightarrow 0^{+}} \Pi_{B}\left(\sigma^{* A}(\varepsilon), x_{1}\right)=\lim _{\varepsilon \rightarrow 0^{+}} \Pi_{B}\left(\sigma^{* A}(\varepsilon), \sigma^{* B}(\varepsilon)\right)<$ $\Pi_{B}\left(\sigma^{* A}(\varepsilon), \sigma^{* B}(0)\right)$. So, $\sigma_{1}^{* A}(\varepsilon) \rightarrow \sigma_{1}^{* A}(0)$ when $\varepsilon \rightarrow 0^{+}$. Now assume that $\lim _{\varepsilon \rightarrow 0^{+}} \sigma_{2}^{* A}(\varepsilon)>\sigma_{2}^{* A}(0)$ (the proof for the $\lim _{\varepsilon \rightarrow 0^{+}} \sigma_{2}^{* A}(\varepsilon)<\sigma_{2}^{* A}(0)$ case is symmetric). Since $\sigma_{1}^{* A}(\varepsilon) \rightarrow \sigma_{1}^{* A}(0)$ when $\varepsilon \rightarrow 0^{+}$it must be the case that $\lim _{\varepsilon \rightarrow 0^{+}} \Pi_{B}\left(\sigma^{* A}(\varepsilon), x_{2}\right)=\lim _{\varepsilon \rightarrow 0^{+}} \Pi_{B}\left(\sigma^{* A}(\varepsilon), \sigma^{* B}(\varepsilon)\right)<\Pi_{B}\left(\sigma^{* A}(\varepsilon), \sigma^{* B}(0)\right)$. So, $\sigma_{2}^{* A}(\varepsilon) \rightarrow \sigma_{2}^{* A}(0)$ when $\varepsilon \rightarrow 0^{+}$. We continue in the same fashion step by step until the last location and we show that $\sigma_{j}^{* A}(\varepsilon) \rightarrow \sigma_{j}^{* A}(0)$ when $\varepsilon \rightarrow 0^{+}$for any $j$. The proof for why $\sigma_{j}^{* B}(\varepsilon) \rightarrow \sigma_{j}^{* B}(0)$ when $\varepsilon \rightarrow 0^{+}$ for any $j$ is equivalent.

Step 3 When $\varepsilon \rightarrow 0^{+}$and when the voter expects that the candidates play $\sigma^{* A}(\varepsilon) \rightarrow \sigma^{* A}(0)$ and $\sigma^{* B}(\varepsilon) \rightarrow \sigma^{* B}(0)$, conditional on being uninformed, the probability that the voter votes for $A$ is $F\left(x_{n / 2}\right)$ when we are in the information set $L$ and $1-F\left(x_{n / 2}\right)$ when we are in the information set $R$.

For any even $n$, when the voter is uninformed and she expects that the candidates chose $\sigma^{* A}(0)$ and $\sigma^{* B}(0)$ we know that:

$$
\begin{aligned}
& E u_{m}\left(\sigma^{* A}(0) \mid L\right)>E u_{m}\left(\sigma^{* B}(0) \mid L\right) \text { and } E u_{m}\left(\sigma^{* B}(0) \mid R\right)>E u_{m}\left(\sigma^{* A}(0) \mid R\right) \text { if } x_{m}<\frac{1}{2} \text { and } \\
& E u_{m}\left(\sigma^{* A}(0) \mid L\right)<E u_{m}\left(\sigma^{* B}(0) \mid L\right) \text { and } E u_{m}\left(\sigma^{* B}(0) \mid R\right)<E u_{m}\left(\sigma^{* A}(0) \mid R\right) \text { if } x_{m}>\frac{1}{2} .
\end{aligned}
$$

By continuity of $E u_{m}\left(\sigma^{Q} \mid S\right)$ in $\sigma^{Q}$ for any $Q \in\{A, B\}$ and $S \in\{L, R, I\}$ it follows that when $\sigma^{*}(\varepsilon) \rightarrow \sigma^{*}(0)$ we should have $E u_{m}\left(\sigma^{* Q}(\varepsilon) \mid S\right) \rightarrow E u_{m}\left(\sigma^{* Q}(0) \mid S\right)$ for any $Q \in\{A, B\}$ and $S \in\{L, R, I\}$. That is, all the above strict inequalities should be maintained for $\varepsilon$ sufficiently small and, thereafter, the probability that the voter votes for a specific candidate conditional on being uninformed should remain unchanged too.

Proof of proposition 7. Notice that when $x_{m} \in\{0,1\}$ then independently of whether the voter is perfectly or imperfectly informed about the policy platforms of the candidates or not, her choice is always the same, that is, even if she is imperfectly informed she votes for the candidate she would have voted for if she had perfect information. To see this consider an imperfectly informed voter with $x_{m}=0$ and assume that we are in $L$. Then the voter knows that the policy candidate $A$ offers gives her a strictly larger utility than the policy candidate $B$ offers and correctly votes for $A$. The voter makes an equivalently correct choice when we are in information set $R$ (she knows that candidate $B$ offers a better policy compared to 
the one offered by $A$ ). And, obviously, she also makes the same choice in both cases when we are in $I$ (she votes for the advantaged candidate $A$ ). So when the voter has imperfect information her behavior may be different from the perfect information case only when $x_{m}=\frac{1}{2}$.

Assume that when the voter is imperfectly informed and $x_{m}=\frac{1}{2}$ then she votes for the advantaged candidate $A$ in any of the three information sets. If this is the case then obviously $p_{L}=p_{R}=2 / 3$.

Assume that an equilibrium in mixed strategies exist for the policy selection subgame in which both candidates place a positive weight at all three policies. Let $\Pi^{A}\left(\sigma^{* A}(\varepsilon), \sigma^{* B}(\varepsilon)\right)$ denote the expected payoff of candidate $A$ when candidates use the assumed mixed strategies and the candidates expect $p_{L}=p_{R}=2 / 3$. Then we should have:

$$
\begin{aligned}
& \cdot \Pi^{A}\left(0, \sigma^{* B}(\varepsilon)\right)=\sigma_{1}^{* B}(\varepsilon)+\sigma_{2}^{* B}(\varepsilon)\left[(1-\varepsilon) \frac{2}{3}+\varepsilon \frac{1}{3}\right]+\sigma_{3}^{* B}(\varepsilon)\left[(1-\varepsilon) \frac{2}{3}+\varepsilon \frac{2}{3}\right] \\
& \left.\cdot \Pi^{A}\left(\frac{1}{2}, \sigma^{* B}(\varepsilon)\right)\right)=\sigma_{1}^{* B}(\varepsilon)\left[(1-\varepsilon) \frac{2}{3}+\varepsilon \frac{2}{3}\right]+\sigma_{2}^{* B}(\varepsilon)+\sigma_{3}^{* B}(\varepsilon)\left[(1-\varepsilon) \frac{2}{3}+\varepsilon \frac{2}{3}\right] \\
& \cdot \Pi^{A}\left(1, \sigma^{* B}(\varepsilon)\right)=\sigma_{1}^{* B}(\varepsilon)\left[(1-\varepsilon) \frac{2}{3}+\varepsilon \frac{2}{3}\right]+\sigma_{2}^{* B}(\varepsilon)\left[(1-\varepsilon) \frac{2}{3}+\varepsilon \frac{1}{3}\right]+\sigma_{3}^{* B}(\varepsilon)
\end{aligned}
$$

and

$$
\begin{aligned}
& \cdot \Pi^{B}\left(\sigma^{* A}(\varepsilon), 0\right)=\sigma_{2}^{* A}(\varepsilon)\left[(1-\varepsilon) \frac{1}{3}+\varepsilon \frac{1}{3}\right]+\sigma_{3}^{* A}(\varepsilon)\left[(1-\varepsilon) \frac{1}{3}+\varepsilon \frac{1}{3}\right] \\
& \cdot \Pi^{B}\left(\sigma^{* A}(\varepsilon), \frac{1}{2}\right)=\sigma_{1}^{* A}(\varepsilon)\left[(1-\varepsilon) \frac{1}{3}+\varepsilon \frac{2}{3}\right]+\sigma_{3}^{* A}(\varepsilon)\left[(1-\varepsilon) \frac{1}{3}+\varepsilon \frac{2}{3}\right] \\
& \cdot \Pi^{B}\left(\sigma^{* A}(\varepsilon), 1\right)=\sigma_{1}^{* A}(\varepsilon)\left[(1-\varepsilon) \frac{1}{3}+\varepsilon \frac{1}{3}\right]+\sigma_{2}^{* A}(\varepsilon)\left[(1-\varepsilon) \frac{1}{3}+\varepsilon \frac{1}{3}\right]
\end{aligned}
$$

In equilibrium we must have that $\Pi^{A}\left(x, \sigma^{* B}(\varepsilon)\right)=\Pi^{A}\left(\sigma^{* A}(\varepsilon), \sigma^{* B}(\varepsilon)\right)$ for all $x$. Moreover we know that $\sigma_{1}^{* A}(\varepsilon)+\sigma_{2}^{* A}(\varepsilon)+\sigma_{3}^{* A}(\varepsilon)=1$ and $\sigma_{1}^{* B}(\varepsilon)+\sigma_{2}^{* B}(\varepsilon)+\sigma_{3}^{* B}(\varepsilon)=1$. We solve the system and we get the unique solution:

$$
\sigma_{1}^{* A}(\varepsilon)=\sigma_{3}^{* A}(\varepsilon)=\frac{1}{3+2 \varepsilon}, \sigma_{2}^{* A}(\varepsilon)=\frac{1+2 \varepsilon}{3+2 \varepsilon} \text { and } \sigma_{1}^{* B}(\varepsilon)=\sigma_{3}^{* B}(\varepsilon)=\frac{1+\varepsilon}{3+2 \varepsilon}, \sigma_{2}^{* B}(\varepsilon)=\frac{1}{3+2 \varepsilon} .
$$

If another equilibrium existed in the policy selection subgame then (using the arguments of the proof of proposition 2) it should be the case that infinitely many mixed equilibria with full support should exist. This would contradict the existence of a unique solution for the system we have just solved.

Thus, the policy selection subgame admits a unique equilibrium. To conclude the proof that the SE that we describe actually exists we only need to argue that when the voter is imperfectly informed and 
$x_{m}=\frac{1}{2}$ she always votes for $A$. Due to the fact that both in $L$ and in $R$ candidate $A$ is more likely to be offering policy $\frac{1}{2}$ than candidate $B$ it is straightforward that she prefers to vote for $A$.

In order to show that this the unique optimal behavior for the voter, suppose that when the voter is imperfectly informed and $x_{m}=\frac{1}{2}$ she does not always vote for $A$. Then, there are a few possibilities: i) she always votes for $B$, ii) she votes for $A$ when we are in $L$ and for $B$ when we are in $R$ and iii) she votes for $B$ when we are in $L$ and for $A$ when we are in $R$. We can repeat the same steps that we performed when we assumed that she always votes for $A$ and we can show that the strategies that the candidates choose in the equilibrium of the policy selection subgame are inconsistent with each of these three new assumptions (cases i), ii) and ii))

Proof of proposition 8 . Consider without loss of generality that $F\left(x_{n / 2}\right) \geq \frac{1}{2}$. First of all, it is straightforward to see that in the conventional advantage game $E\left[u_{m}\left(x_{A}\right): b_{I\left(x_{A}, x_{B}\right)}\right]>E\left[u_{m}\left(x_{B}\right): b_{I\left(x_{A}, x_{B}\right)}\right]$ for any $b_{I\left(x_{A}, x_{B}\right)}$ and any $d>0$. That is the voter always votes for $A$ when we are in information set $I$. Now, let's define $\mu=\min \left\{\min \left\{\phi\left(\left|x_{i}-x_{1}\right|\right)-\phi\left(\left|x_{i+1}-x_{1}\right|\right) \mid i=1,2, \ldots, n-1\right\} \cup \min \left\{\phi\left(\left|x_{i+1}-x_{n}\right|\right)-\right.\right.$ $\left.\left.\phi\left(\left|x_{i}-x_{n}\right|\right) \mid i=1,2, \ldots, n-1\right\}\right\}$. It is clear that for any strictly decreasing $\phi(\cdot)$ it must be the case that $\mu>0$. This implies that if $x_{m}=x_{1}$ and $d \in(0, \mu)$ the voter will vote for $B$ in information set $R$ and for $A$ in information set $L$ for any belief system $\left(b_{L}, b_{I}, b_{R}\right)$. Since $F$ has full support all the above suggest that $\left(p_{L}, p_{R}\right) \in\left(\min \left\{f\left(x_{1}\right), f\left(x_{n}\right)\right\}, 1-\min \left\{f\left(x_{1}\right), f\left(x_{n}\right)\right\}\right)^{2}$ and $p_{I}=1$ when $d \in(0, \mu)$. By the arguments of the proof of proposition 1 it is clear that for any belief system which induces $\left(p_{L}, p_{R}\right) \in\left(\min \left\{f\left(x_{1}\right), f\left(x_{n}\right)\right\}, 1-\min \left\{f\left(x_{1}\right), f\left(x_{n}\right)\right\}\right)^{2}$ and $p_{I}=1$ there is a unique equilibrium in the candidates' reduced game. Now since both $p_{L}$ and $p_{R}$ are bounded from below by a strictly positive number and from above by a positive number strictly lower than one it must be the case that for any such $p_{L}$ and $p_{R}$ the unique equilibrium of the reduced game is such that, for some $d>0$, when $x_{m}<\frac{1}{2}$ we must have:

$$
E \phi\left(\left|x_{A}-x_{m}\right| \mid L\right)-E \phi\left(\left|x_{B}-x_{m}\right| \mid L\right)>d \text { and } E \phi\left(\left|x_{B}-x_{m}\right| \mid R\right)-E \phi\left(\left|x_{A}-x_{m}\right| \mid R\right)>d
$$

and when $x_{m}>\frac{1}{2}$ we must have:

$$
\begin{aligned}
& E \phi\left(\left|x_{B}-x_{m}\right| \mid L\right)-E \phi\left(\left|x_{A}-x_{m}\right| \mid L\right)>d \\
& E \phi\left(\left|x_{A}-x_{m}\right| \mid R\right)-E \phi\left(\left|x_{B}-x_{m}\right| \mid R\right)>d .
\end{aligned}
$$

Hence, by $E u_{m}\left(\sigma^{* A} \mid S\right)=E \phi\left(\left|x_{A}-x_{m}\right| \mid S\right)+d$ and by continuity of $E u_{m}\left(\sigma^{* A} \mid S\right)$ in $d$ it directly follows that there exists $\hat{d}(n, F)>0$ such that for every $d \in(0, \hat{d}(n, F)]$ any belief system $\left(b_{L}, b_{I}, b_{R}\right)$ 
induces a unique equilibrium in the reduced game which in turn induces $p_{L}=1-p_{R}=F\left(x_{n / 2}\right)$. That is, for sufficiently low values of the advantage the conventional advantage game admits a unique SE which coincides with the unique SE of our game for $\rho=1$.

\section{Appendix 2 (odd number of policies)}

When $n$ is odd, one obtains essentially the same results with the following modifications. The relevant threshold of $\rho \in\left[\frac{1}{2}, 1\right]$ which determines whether we are in the pure strategy equilibrium case or in the mixed strategy no longer relates to $F\left(x_{n / 2}\right)$ but to $F\left(x_{\frac{n-1}{2}}\right)$ and to $1-F\left(x_{\frac{n+1}{2}}\right)$ instead.

A) For $F\left(x_{\frac{n-1}{2}}\right)>1-F\left(x_{\frac{n+1}{2}}\right)$ we have that:

A.1) if $\rho \leq F\left(x_{\frac{n-1}{2}}\right)+\rho f\left(x_{\frac{n+1}{2}}\right)$ then there exists an essentially unique undominated SE in pure strategies, such that both candidates converge to $x=0$.

A.2) if $\rho>F\left(x_{\frac{n-1}{2}}\right)+\rho f\left(x_{\frac{n+1}{2}}\right)$ then there exists a unique undominated SE in mixed strategies, such that the advantaged candidate assigns most of its probability to $x=0$ and the disadvantaged candidate concentrates on $x=1$. In specific both candidates mix according to the strategies presented in proposition 3 with the modification that in this case $H^{*}=\frac{\rho-F\left(x_{\frac{n-1}{2}}\right)-\rho f\left(x_{\frac{n+1}{2}}\right)}{\rho-1+F\left(x_{\frac{n+1}{2}}\right)-\rho f\left(x_{\frac{n+1}{2}}\right)}<1$.

B) For $F\left(x_{\frac{n-1}{2}}\right)<1-F\left(x_{\frac{n+1}{2}}\right)$ we have that:

B.1) if $\rho \leq 1-F\left(x_{\frac{n+1}{2}}\right)+\rho f\left(x_{\frac{n+1}{2}}\right)$ then there exists an essentially unique undominated SE in pure strategies, such that both candidates converge to $x=1$.

B.2) if $\rho>1-F\left(x_{\frac{n+1}{2}}\right)+\rho f\left(x_{\frac{n+1}{2}}\right)$ then there exists a unique undominated SE in mixed strategies, such that the advantaged candidate assigns most of its probability to $x=1$ and the disadvantaged candidate concentrates on $x=0$. In specific both candidates mix according to the strategies presented in proposition 3 with the modification that in this case $H^{*}=\frac{\rho-F\left(x_{\frac{n-1}{2}}\right)-\rho f\left(x_{\frac{n+1}{2}}\right)}{\rho-1+F\left(x_{\frac{n+1}{2}}\right)-\rho f\left(x_{\frac{n+1}{2}}\right)}>1$.

C) Finally suppose that $F\left(x_{\frac{n-1}{2}}\right)=1-F\left(x_{\frac{n+1}{2}}\right)$. In this case, we have that $\rho \geq F\left(x_{\frac{n-1}{2}}\right)+\rho f\left(x_{\frac{n+1}{2}}\right)$ must always hold because $\rho>\frac{1}{2}$ implies $\rho\left(1-f\left(x_{\frac{n+1}{2}}\right)\right)>F\left(x_{\frac{n-1}{2}}\right)$, thus we have that.

C.1) if $\rho=F\left(x_{\frac{n-1}{2}}\right)+\rho f\left(x_{\frac{n+1}{2}}\right)$ then there is no undominated SE (notice that this can only hold for $\left.\rho=\frac{1}{2}\right)$ 
C.2) if $\rho>F\left(x_{\frac{n-1}{2}}\right)+\rho f\left(x_{\frac{n+1}{2}}\right)$ there exists a unique undominated SE in which candidates mix uniformly exactly as in proposition 4 . 


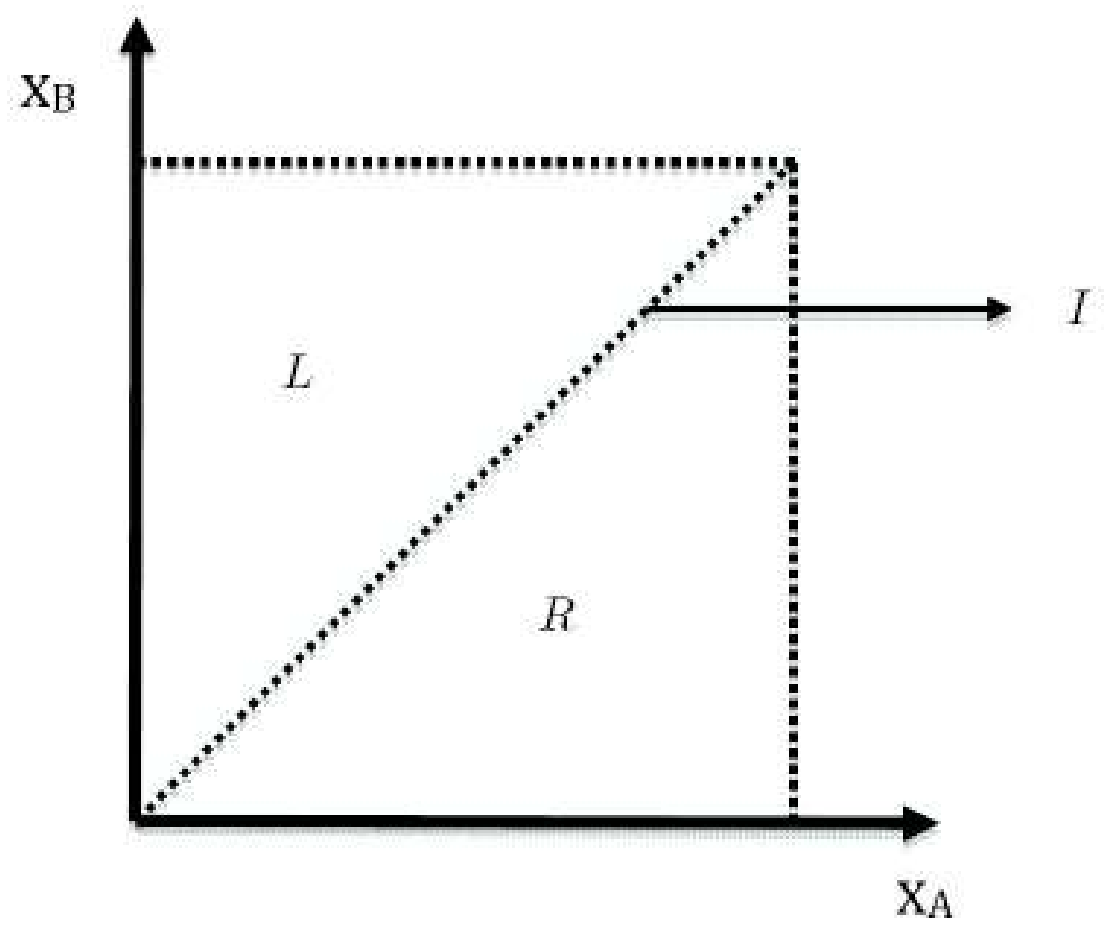

Figure 1a. Partition induced by the signals.



Figure $1 \mathrm{~b}$. Beliefs corresponding to different signals. 


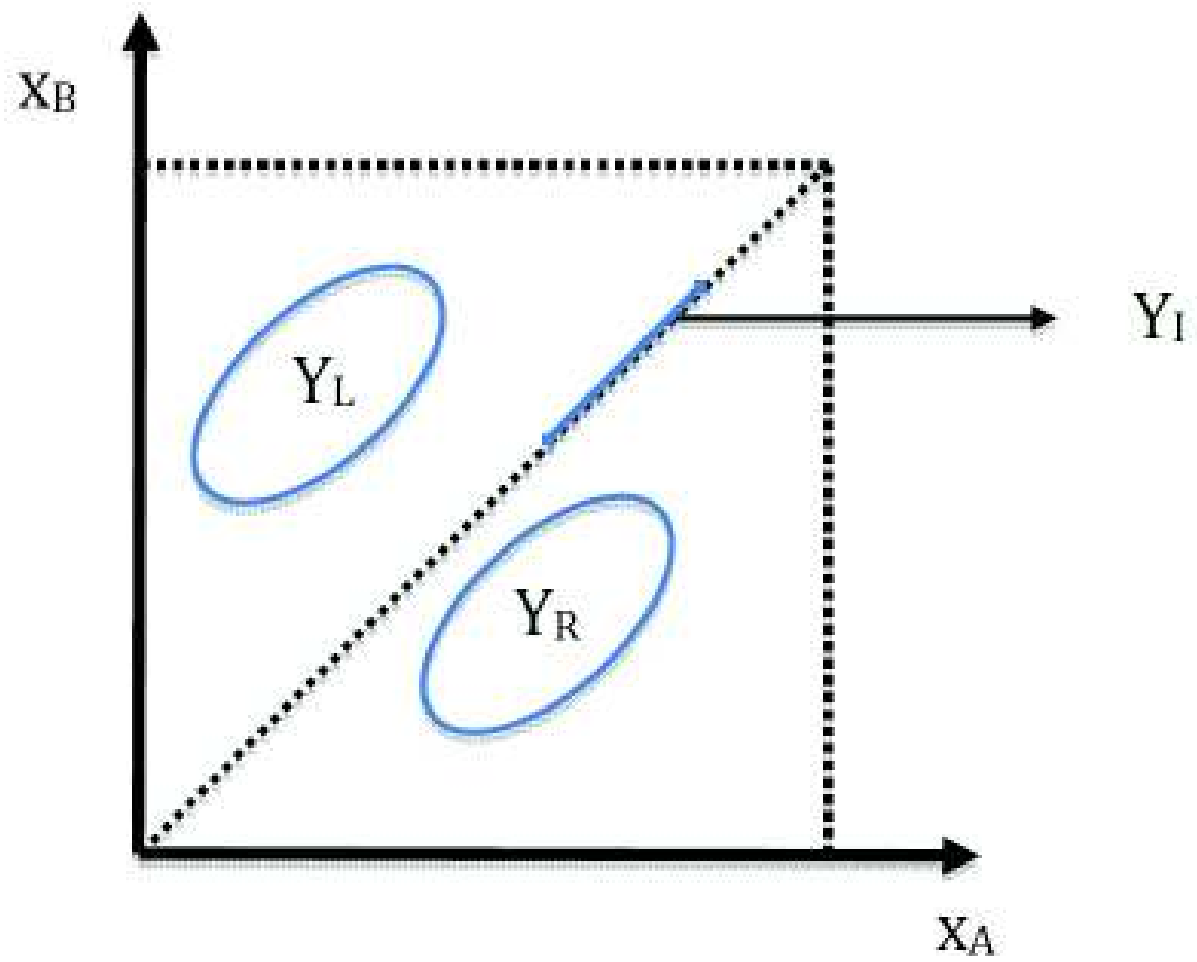

Figure 1c. Example of support of beliefs for different signals. 


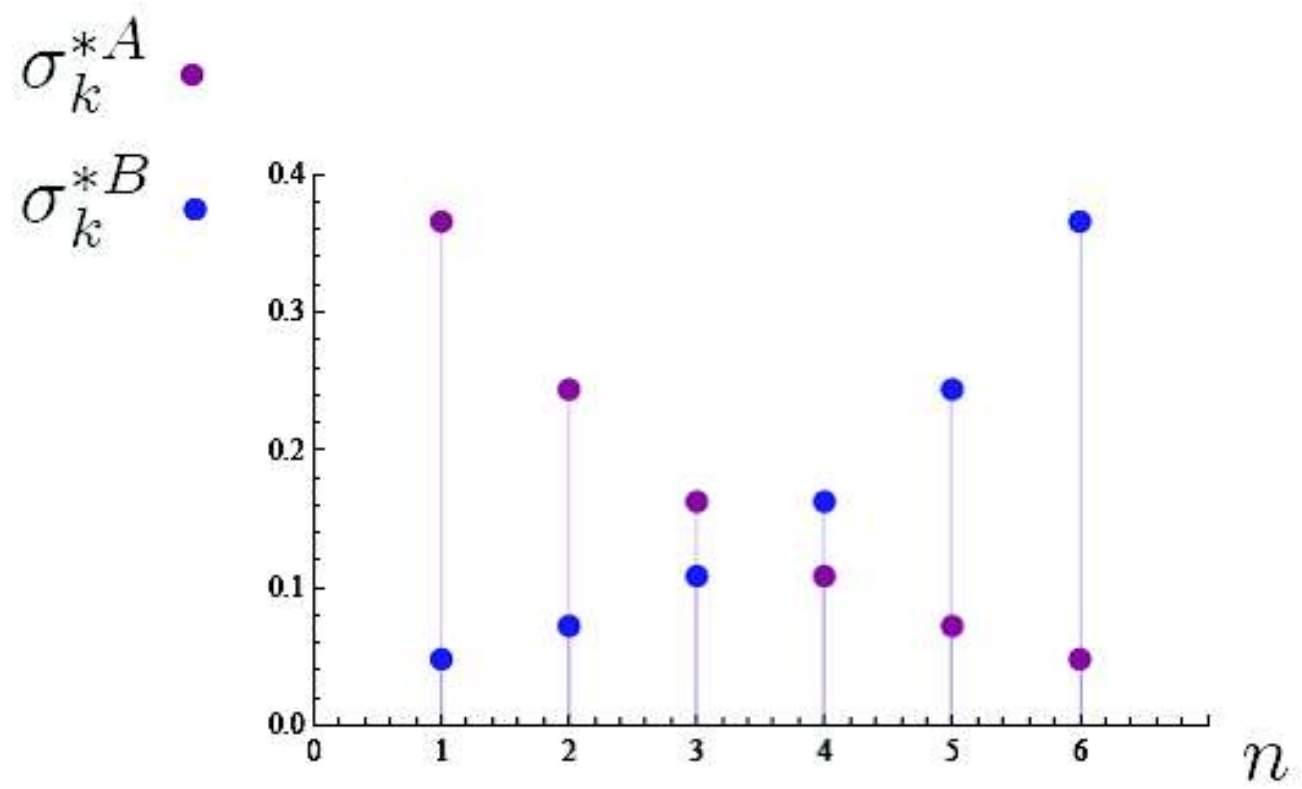

Figure 2a. Equilibrium mixed strategies for $F\left(x_{n / 2}\right)=0.6, \rho=1$ and $n=6$.

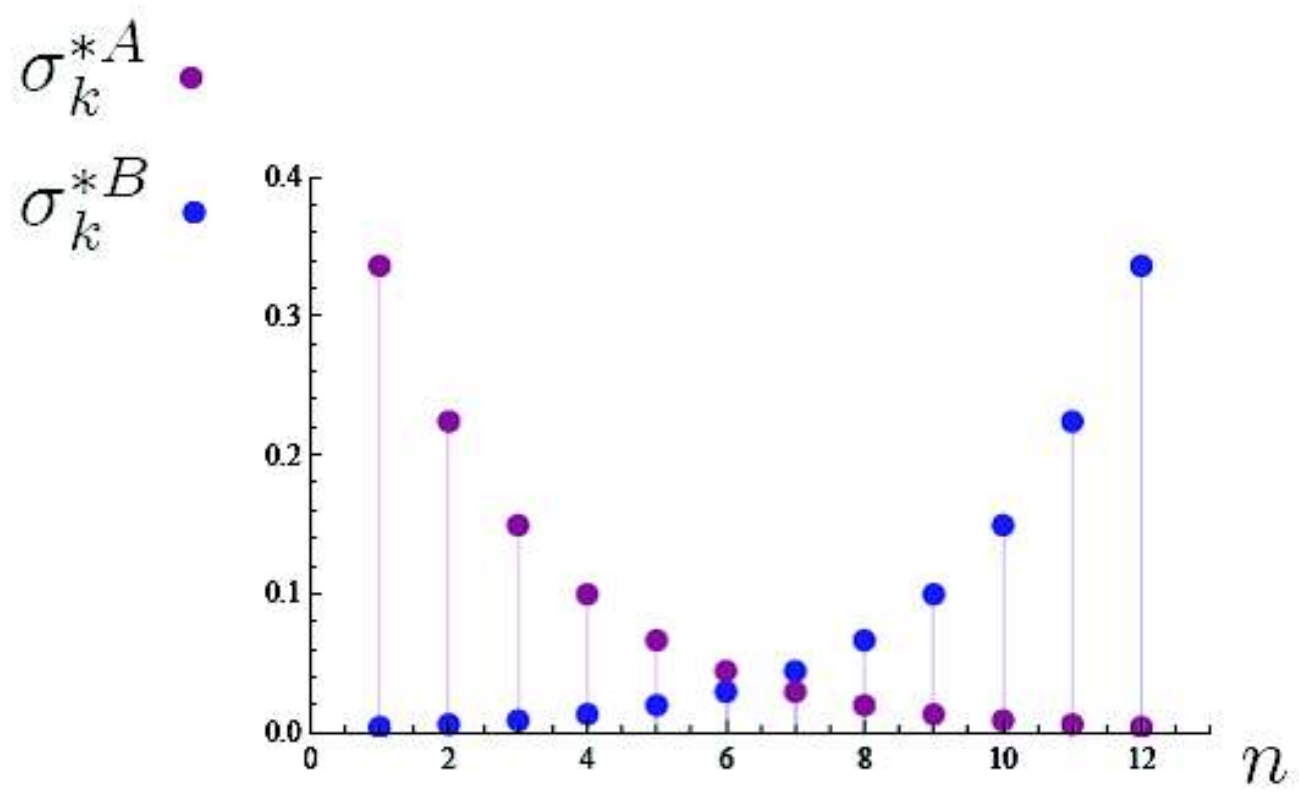

Figure 2b. Equilibrium mixed strategies for $F\left(x_{n / 2}\right)=0.6, \rho=1$ and $n=12$. 


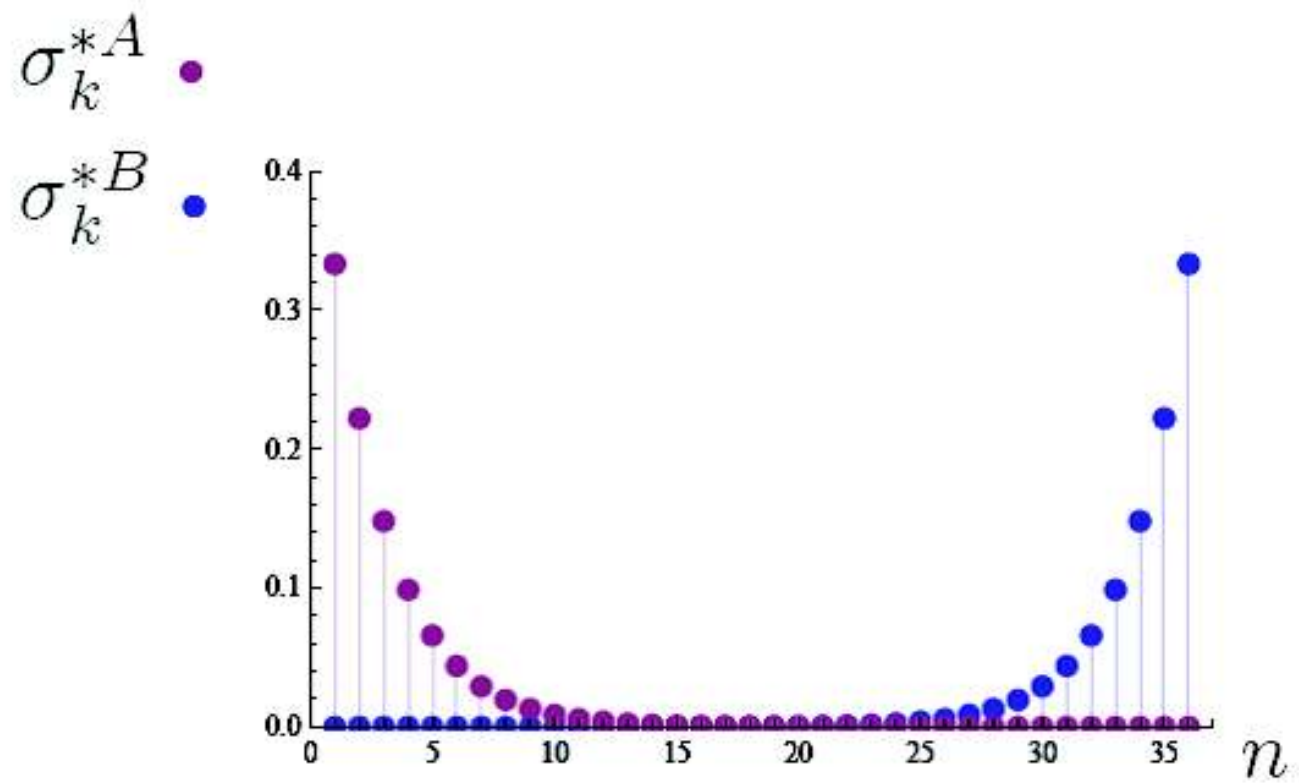

Figure 2c. Equilibrium mixed strategies for $F\left(x_{n / 2}\right)=0.6, \rho=1$ and $n=36$.

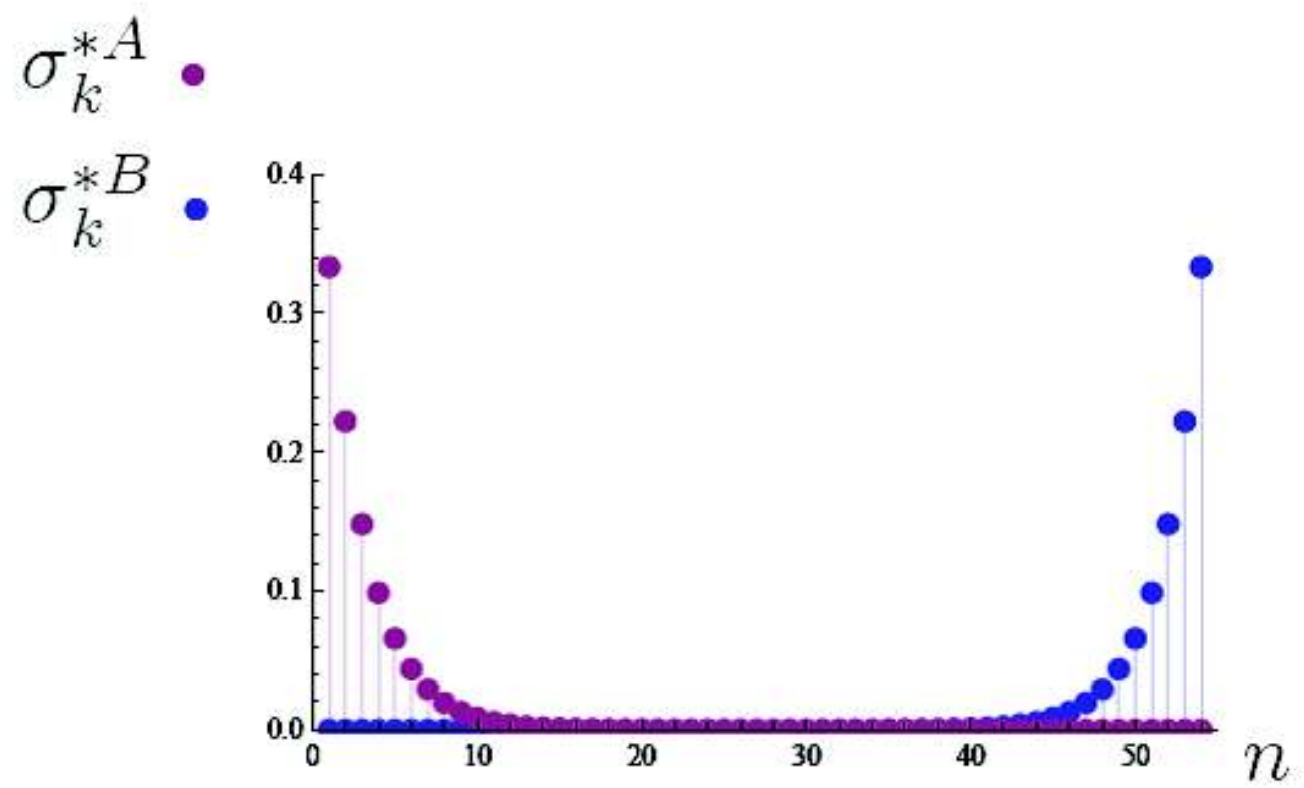

Figure 2d. Equilibrium mixed strategies for $F\left(x_{n / 2}\right)=0.6, \rho=1$ and $n=54$. 




Figure 3a. Equilibrium payoff of candidate $A$ as a function of $\rho$ for $n=6$ and $F\left(x_{n / 2}\right)=0.6$.

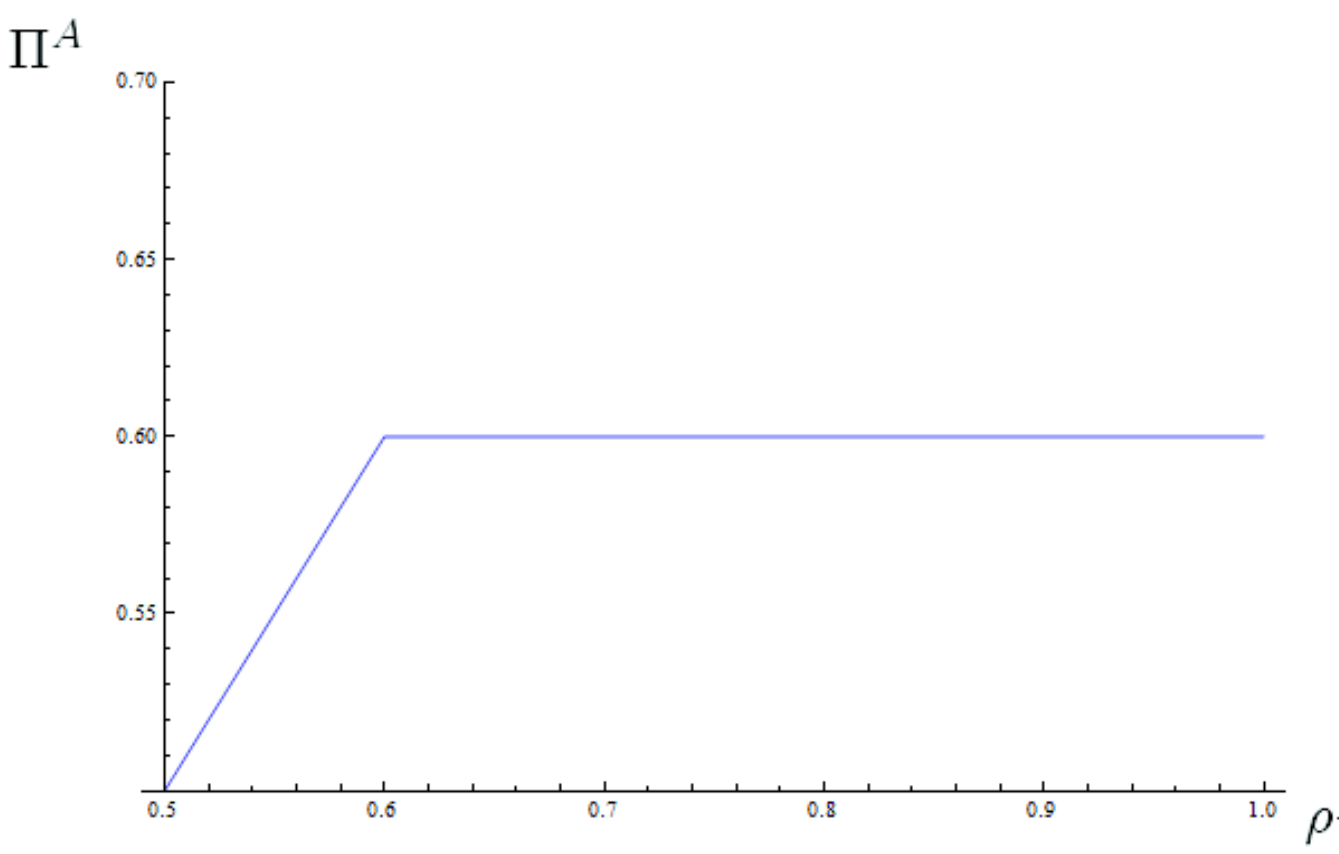

Figure 3b. Equilibrium payoff of candidate $A$ as a function of $\rho$ for $n=200$ and $F\left(x_{n / 2}\right)=0.6$. 


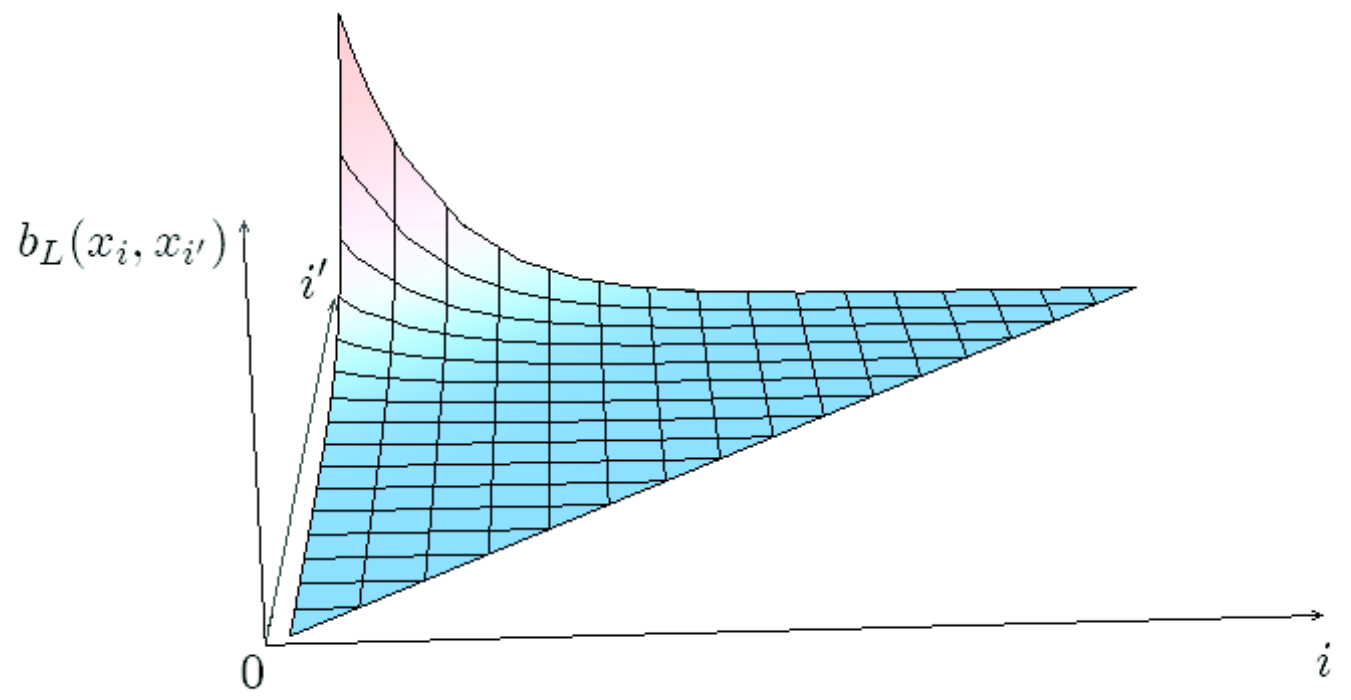

Figure 4a. Equilibrium beliefs for information set $L$ for $F\left(x_{n / 2}\right)=0.6, \rho=1$ and $n=10$.

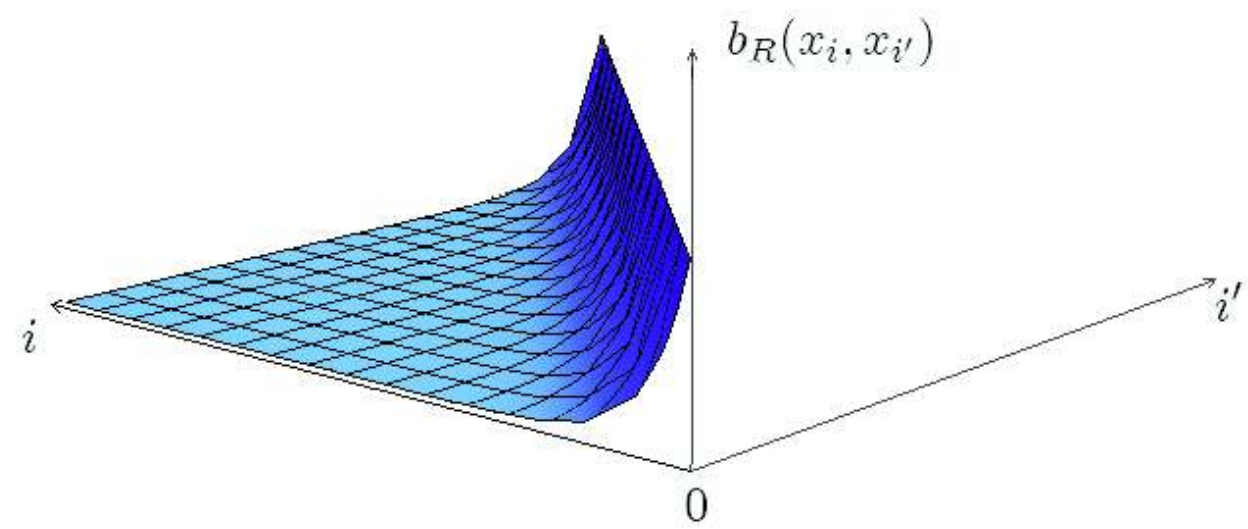

Figure $4 \mathrm{~b}$. Equilibrium beliefs for information set $R$ for $F\left(x_{n / 2}\right)=0.6, \rho=1$ and $n=10$. 